\title{
Fusion of the N-terminal 119 amino acids with the RelA-CTD renders its growth inhibitory effects ppGpp-dependent
}

\author{
Krishma Tailor ${ }^{1,2}$, Prarthi Sagar ${ }^{1}$, Keyur Dave $^{1}$, and Jayashree Pohnerkar ${ }^{1} \S$ \\ ${ }^{1}$ Department of Biochemistry, Faculty of Science, Maharaja Sayajirao University of Baroda, \\ Vadodara - 390002, Gujarat, India. $\square$ \\ ${ }^{2} \square$ Department of Biochemistry and Molecular Biology, Howard University College of \\ Medicine, Washington, DC, U.S.A. \\ Running title: A regulatory function for HD domain of RelA?
}

Key Words: (p)ppGpp, RelA, HD domain, RelA-CTD

$\S$ Corresponding author mailing address: Department of Biochemistry, Faculty of Science, Maharaja Sayajirao University of Baroda, Vadodara - 390002, Gujarat, India.

Phone: +912652795594. E-mail: jayashreepohnerkar@ hotmail.com $\square$

\begin{abstract}
:
The $\square$ guanosine $\square$ nucleotide $\quad$ derivatives $\square$ ppGpp $\square$ and $\square$ pppGpp, are central to the remarkable capacity of bacteria to adapt to fluctuating environment and $\square$ metabolic perturbations. These $\square$ alarmones $\square$ are $\square$ synthesized $\quad$ by two proteins, $\square \operatorname{RelA} \square$ and $\square$ SpoT $\square$ in $\square E$. coli $\square$ and the activities of each of the two enzymes are highly regulated for homeostatic control of (p)ppGpp $\square$ levels in the cell. Although the domain structure and function of RelA are well defined, the findings of this study unfold the regulatory aspect of RelA that is possibly relevant in vivo. We uncover here the importance of the N-terminal 1-119 amino acids of the enzymatically compromised (p)ppGpp hydrolytic domain (HD) of monofunctional RelA for the (p)ppGpp mediated regulation of RelA-CTD function. We find that even moderate level expression of RelA appreciably reduces growth when the basal levels of $\square$ (p)ppGpp $\square$ in the cells are $\square$ higher than in the wild type, an effect independent of its ability to synthesize $\square$ (p)ppGpp. This is evidenced by the growth inhibitory effects $\square$ of $\square \square$ oversynthesis $\square$ of the $\square$ RelA-CTD in the $\square$ relA $\square$ strain but not in $\square$ relA $\square$ null mutant, suggesting the requirement of the functional $\square \operatorname{RelA} \square$ protein for
\end{abstract}


basal level synthesis of $\square$ (p)ppGpp, accordingly corroborated by the $\square$ restoration of the growth inhibitory effects of $\quad$ the RelA-CTD $\square \quad$ expression $\quad$ in the $\square$ relAl spoT202 $\square$ mutant. $\square \square$ The N-terminal 119 amino acids of $\square$ RelA $\square$ fused in-frame with the $\square$ RelA-CTD, $\square$ both from 406-744 amino acids (including TGS) and from 454-744 amino acids (sans TGS) caused growth inhibition only in spoT1 and spoT202 relA1 mutants, uncovering the hitherto unrealized $\square$ (p)ppGpp-dependent regulation of RelACTD function. $\square$ An $\square$ incremental rise in the $\square$ (p)ppGpp $\square$ levels is proposed to progressively modulate the interaction of $\square$ RelA-CTD $\square$ with the $\square$ ribosomes, $\square$ with possible implications in the feedback regulation of $\square$ the N-terminal $\square$ (p)ppGpp $\square$ synthesis function, a proposal that best explains the nonlinear relationship between $\square$ (p)ppGpp $\square$ synthesis and increased ratio of $\square$ RelA:ribosomes, both $\square$ in vitro $\square$ as well as $\square$ in vivo. $\square$ ?

\section{Introduction:}

Environmental stressors elicit largely conserved adaptive responses in bacteria (and plants), mediated and coordinated by the hyperphosphorylated nucleotides, ppGpp and pppGpp (together called (p)ppGpp). These $\square$ signalling $\square$ nucleotides $\square$ control various cellular activities at transcriptional, translational, and posttranslational levels (Hauryliuk et al., 2015). Recent studies have realized that the intracellular pool size of $\square$ (p)ppGpp $\square$ does not act as a biphasic switch $\square$ rather, incremental levels of $\square$ (p)ppGpp $\square$ exert differential effects on cell physiology including its role in virulence, pathogenesis, antibiotic resistance/tolerance, sporulation, biofilm $\square$ and $\square$ persisters' cell formation (Dozot et al., 2006, Geiger et al., 2010, Ochi et al., 1981, Poole, 2012, Schofield et al., 2018); this is besides the well characterised stringent response (Cashel et al., 1996, Potrykus \& Cashel, 2008) $\square$.

These nucleotides $\square$ derivatives $\square$ are metabolised $\square$ by two types of proteins - widely distributed, highly conserved, long multidomain, bifunctional RSH (RelA SpoT Homolog) and monofunctional small alarmone synthetases (SASs) and hudrolase (SAHs) which are 
limited in their distribution to Gram positive $\square$ Firmicutes $\square$ and $\square$ Actinobacteria (Atkinson et al., 2011, Gaca et al., 2015, Ronneau \& Hallez, 2019, Steinchen \& Bange, 2016) $\square . \square \operatorname{In} \square E$. $\square$ coli $\square$ and $\quad$ in $\quad$ other $\square \gamma$-proteobacteria, $\square$ (p)ppGpp $\square$ is $\quad$ synthesised by long $\square$ monofunctional $\square$ RSH, RelA, whose hydrolytic domain is heavily compromised; whereas, the second RSH protein, SpoT is a bifunctional enzyme containing both synthetase and hydrolase activities and normally function in homeostatic regulation of (p)ppGpp levels by hydrolysing excess (p)ppGpp (An et al., 1979, Hernandez \& Bremer, 1991). SpoT is nevertheless a weak (p)ppGpp synthetase, responsive to signals of fatty acid, iron and carbon limitation $\square$ (Iyer et al., 2018, Seyfzadeh et al., 1993, Spira et al., 1995, Vinella et al., 2005, Wang et al., 2016, Xiao et al., 1991); $\square$ interestingly though, fatty acid starvation induced rapid synthesis of $\square$ (p)ppGpp $\square$ has recently been shown to lead in part to $\square$ amino $\square$ acid starvation $\square$ and contribution $\square$ from $\square \operatorname{RelA} \square$ followed by its delayed synthesis by SpoT (Gentry \& Cashel, 1996, Sinha et al., 2019). The $\square$ characteristic functional elements of the long RSH enzyme (RelA/SpoT $\square$ and $\square$ Rel) includes a N-terminal enzymatic domain harbouring motif for $\square$ hydrolase $\square$ and/or $\square$ (p)ppGpp $\square$ synthetase $\square$ activity and a C-terminal regulatory domain (CTD) containing conserved motifs $-\square$ TGS (ThrRS, $\square$ GTPases $\square$ and $\square$ SpoT), $\quad$ DC $\quad$ (aspartate-cysteine motif) and ACT (aspartate $\square$ kinase, $\square$ chorismate $\square$ mutase $\square$ and $\square$ TyrA) also called RRM (RNA recognition motif) $\square$ (Atkinson et al., 2011). $\square$ Basic regulation $\square$ of $\square \square$ (p)ppGpp $\square$ synthesis function of long RSH enzymes is quite well conserved across mono- and $\square$ bifunctional $\square$ long RSH proteins which is reproduced $\square$ in vitro $\square$ (Arenz et al., 2016, Brown et al., 2016, Haseltine \& Block, 1973) (Kushwaha et al., 2019, Loveland et al., 2016, Winther et al., 2018). $\square \square \square$ The CTD of the long RSH proteins has $\square \mathrm{a} \square$ unique regulatory role $\square$ of inhibiting the $\square$ (p)ppGpp $\square$ synthetase $\square$ function of the NTD but not the hydrolytic activity by the conserved aspartate-cysteine motif (DC) that has been $\square$ also proposed to be involved in $\square$ the 
oligomerization $\square$ of $\square$ RelA $\square$ in the ribosome unbound state $\square$ (Gratani et al., 2018, Yang \& Ishiguro, 2001, Avarbock et al., 2005, Butland et al., 2008, Gropp et al., 2001); $\square$ the activation of ribosome-bound $\square \operatorname{RelA} \square$ is $\square$ proposed to be due to $\square$ the association of $\square$ monomeric $\square$ RelA $\square$ with $\quad$ the $\square$ ribosome $\square$ and $\square$ deacylated $\square$ tRNA $\square$ which $\square$ relieves the $\square$ autoinhibitory $\square$ effect of the CTD on the $\square$ NTD $\square$ (Arenz et al., 2016) $\square$ (Avarbock et al., 2005) $\square$ (Jain et al., 2006b, Loveland et al., 2016). $\square$ Furthermore, recent studies indicated $\square$ an $\square$ allosteric regulation of $\square$ RelA, both positive and negative by the end product, (p)ppGpp. $\square$ The regulatory effectors of $\square$ RelA $\square$ enzymatic activity for $\square$ (p)ppGpp $\square$ synthesis include positive end product activation by $\square \operatorname{pppGpp} \square \square$ at low $(\sim 100 \mathrm{uM})$, and negative inhibitory effect at higher $(\sim 400 \square \mathrm{uM})$ concentration $\square$ (Kudrin et al., 2018, Wendrich et al., 2002, Shyp et al., 2012); the site for positive activation has been localized to the N-terminal segment (NTD) segment $\quad$ of $\square \operatorname{Rel} \square$ of $\quad \square$ B. $\quad$ subtilis, $\square \square S . \quad$ equisimilis, $\quad M$. tuberculosis, $\square$ M. $\square$ smegmatis $\square$ and $\square$ Francisella $\square$ tularensis (Jain et al., 2006a, Wilkinson et al., 2015, Takada et al., 2021) and $\square \operatorname{RelA} \square$ of $\square$ Escherichia $\square$ coli $\square($ Kudrin et al., 2018, Shyp et al., 2012, Takada et al, 2021). $\square$ Interestingly, $\square($ p)ppGpp-mediated regulation of synthase $\square$ activity $\quad$ of $\square$ Rel $\square$ by $\quad$ the $\square$ Rel-CTD $\square$ in $\square$ M. $\square$ smegmatis $\square$ has $\square$ also $\square$ been demonstrated. $\square$ The mechanism of negative feedback regulation $\square$ has been proposed to $\square$ be important for fine tuning the $\square$ Rel's $\square$ (p)ppGpp $\square$ synthesis function (Syal et al., 2015). $\square$

The results presented here $\square$ provide evidence for the existence of regulation by $\square$ (p)ppGpp $\square$ of the RelA-CTD $\square$ function in $\square E$. $\square$ coli. $\square \square$ The novel aspect of regulation of $\square$ RelA's $\square$ growth inhibitory function became apparent with the fortuitous cloning of $\square$ RelA-CTD sequence remaining after the removal of the DNA between two $\square P v u I I \square$ sites at positions 354 and 1362 in the relA gene sequence which also results in an in-frame fusion of the N-terminal 1- 119 amino acids to 454-744 amino acid sequence of the RelA-CTD. We showed that $\square$ the $\square$ low to moderate level 
expression $\square$ of $\square$ RelA-CTD fusion gene significantly inhibits growth, albeit only when the basal levels of $\square$ (p)ppGpp $\square$ are higher than in the wild type. $\square \square$ Thus, the combination of mutations, $\square$ relA $A^{+} \square$ spoT1 $\square$ and $\square$ relAl spoT202 $\square$ which enhances intracellular levels of (p)ppGpp more than that by relAl spoTl or relA $^{+}$spoT $^{+}$, was $\square$ effective in $\square$ promoting inhibition $\square$ of the growth by fusion RelA-CTD, $\square$ substantiating $\square$ the growth inhibitory effects $\square$ to be $\square$ dictated by the intracellular levels of (p)ppGpp and independent of RelA's $\square$ (p)ppGpp $\square$ synthesis ability. This result was subsequently confirmed by PCR based in-frame fusion of 1-357 nucleotides (119 amino acids) to 1014-2235 nucleotides of RelA-CTD (406-744 amino acids) which additionally contains TGS domain. $\square \square$ Our $\square$ results $\square$ are compatible with the proposal that (p)ppGpp $\square \square$ mediates, through the N-terminal 119 amino acids, regulation of binding of RelA-CTD $\square$ to the target ribosomes, $\square$ presumably important in the feedback regulation of RelA's (p)ppGpp synthesis function. Recently, very high-level expression of RelA-CTD has been shown to inhibit the growth, irrespective of the $\square$ relA $\square$ genotype of the host as a result of the decrease in the rate of protein synthesis and interference in the normal stringent response (Gropp et al., 2001), whereas $\square$ moderate levels are without an effect on growth (Turnbull et al., 2019). $\square$ The significance of our results is discussed. $\square$

\section{Results:}

The genesis of the present work was an attempt to test the functional significance of the synteny of relA (encoding (p)ppGpp synthetase I) and rumA (coding for 23S rRNA methyl transferase) in genomes of several gram-negative bacteria, including some important pathogens. Incidentally, the U1939 of 23S rRNA is placed close to the acceptor arm of the uncharged tRNA where RelA binds on the ribosome (Yusupov et al., 2001, Persaud et al., 
2010). Persaud et.al., (2010) addressed the functionality of rumA and reported rumA to be inessential for growth as the null mutation in the rumA gene is not associated with any growth defect. Since their mutation in rumA was a deletion of its ORF, and since all the promoters of $\mathrm{relA}$ are present in the upstream rumA gene (Fig.1), both transcriptional and translational regulation of $\mathrm{relA}$, if there were any, are expected to be abolished in the mutant. We tested the essentiality of rumA in this work by instead creating an insertion of chloramphenicol acetyl transferase $(C A T)$ or Kanamycin resistance $(K A N)$ cassette in the rumA ORF at a unique SalI site -878 bp upstream of all the known promoters of relA gene (Fig.1). Interestingly, we find that the CAT insertion in one of the two orientations exerted a cis effect on expression of relA gene, causing elevated synthesis of RelA protein, whereas, insertion of the $C A T$ gene in the opposite orientation, and the insertion of $K A N$ gene cassette in either orientation, did not exert any effect on expression of relA (see below), validating the result that lack of methylation at U1939 of 23SrRNA by RumA is not relevant for relA regulation (Persaud et al., 2010). We investigated in this paper the cis effect of the CAT insertion mutation that increases relA expression and its phenotypic consequences.

Because of the intrinsic variation in the basal levels of (p)ppGpp in several Escherichia coli strains due to polymorphism at relA and spoT (Laffler \& Gallant, 1974a, Brown et al., 2002, King et al., 2004, Spira \& Ferenci, 2008, Ferenci et al., 2011), we used two representative strains of $E$. coli, namely MG1655 and MC4100 which are recognized as being (p)ppGpp sensitive and tolerant respectively, in terms of (p)ppGpp related phenotypes being accentuated in the latter strain. We performed most of the experiments with both the strains and found a large part of the results to be same between them. To avoid repetition, the results described here are reported for the strain MG1655 unless indicated otherwise. Briefly, genotypes relevant to (p)ppGpp homeostasis for each of the strain are: MG1655 is wildtype at 
both relA and spoT loci, whereas MC4100 has two mutations, relAl and spoTl. The relAl mutation is an IS 2 insertion between $85^{\text {th }}$ and $86^{\text {th }}$ codon of relA gene, as a result, the mutant protein retains $1 \%$ residual RelA activity (Metzger et al., 1989). The spoTl allele contains a substitution $(\mathrm{H} 255 \mathrm{Y})$ in the synthesis domain and a two-amino acid insertion between residues 82 and 83(+QD) in the hydrolysis domain of the SpoT protein (Spira et al., 2008). The compromised (p)ppGpp synthetic and hydrolytic functions of SpoT1 are responsible for slow (20-fold) decay in the first order kinetics of (p)ppGpp during stringent response as well as a severe impairment in (p)ppGpp degradation during exponential growth, resulting in its higher basal levels (Laffler \& Gallant, 1974a, Laffler \& Gallant, 1974b, Fiil et al., 1977, Sarubbi et al., 1988). However, the reasons for sensitivity and tolerance of strains MG1655 and MC4100 respectively to (p)ppGpp mediated effects are more than the differences at relA and spoT (Spira \& Ferenci, 2008).

\section{Insertion of $C A T / K A N$ cassette in $\square$ rum $A$ gene $\square$}

rumA mutants were generated by the strategy of recombineering (Datsenko \& Wanner, 2000) of $C A T / K A N$ cassette in rumA DNA. (i) $C A T$ cassette was inserted at the unique SalI restriction enzyme site -878 bp upstream of relA sequence in the orientation where the direction of transcription/translation of CAT is same as that of relA in the MG1655/MC4100 relA ${ }^{+}$background. This insertion is upstream of all the known promoters of relA mapped in rumA gene sequence (Fig.1), and the only one to affect the growth phenotype of the mutant (see below). (ii) The CAT insertion at SalI restriction enzyme site in the opposite orientation in KP59 and KP60 (Fig.3) or (iii) in either orientation at the unique $M l u I$ restriction enzyme site $(-1310$ bp) in rumA DNA (KP9, KP25) did not have any effect on growth (and relA synthesis) (Fig.3). (iv) We next tested if CAT gene replacement by $K A N$ gene cassette affects growth if its direction of transcription/translation were same as that of the relA. The $K A N^{\mathrm{r}}$ cassette was cloned at Sall site in either orientation in the 
rumA DNA by the recombineering method same as that for the CAT gene (see Materials and Methods). However, the $K A N^{\mathrm{r}}$ insertion was without any effect on the growth/expression of RelA (data not shown). The phenotypic effects of orientation specific insertion of CAT gene in $\operatorname{rum} A$ can be due to the chance generation of a promoter at the unique junction between the $C A T$ and rumA sequences. We did not study this proposal further.

We describe below the phenotypes of the effect of increased synthesis of $\square$ RelA $\square$ in KP32 (spoT1 rumASalI::CAT). The insertion mutation in rumA is hereafter referred to as rumA::CAT.

The requirement of spoT1 mutation is obligatory for manifestation of the $\operatorname{rumA}: \mathrm{CAT}$ phenotype

The fact that spoT1 mutation is required for the phenotype became evident when the effect of the mutation was manifest in KP4 (MC4100 relA ${ }^{+}$spoT1) but not in MG1655 strain. We constructed spoT1 derivative of MG1655 by cotransduction with linked $\triangle p y r E 748:: K A N$. P1 lysate prepared on KP11 (MC4100 spoT1 1 pyrE748::KAN) was used to transduce spoT1 mutation in MG1655 strain. The spoT1 mutation was verified by DNA sequencing (data not shown). In contrast to KP4 (MC4100 relA spoT1) strain, MG1655 spoT1 transductant (KP31) was marginally affected for growth in minimal medium (Fig.2A) (Sarubbi et al., 1988).

\section{Phenotypes of $\square$ overexpression $\square$ of $\square$ relA:}

(i) The $\operatorname{rum} A:$ :CAT mutant (KP32) grows unusually slowly on M9 minimal medium

Of the two important phenotypes of the rumA::CAT mutant described here, the first pertains to the growth of the mutant on M9 minimal agar plate. The slow growth of KP32 (spoT1 $\operatorname{rumA::CAT)}$ is striking in that there is no colony formation for $\sim 24$ hours of incubation on minimal medium at $37^{\circ} \mathrm{C}$ (Fig.2A). This slow growth phenotype was confirmed by measuring steady state growth rates of different mutants (Fig.2B). Following nutrient shift 
down from LB to M9 broth, KP32 (spoT1 rumA::CAT) mutant reproducibly exhibited a long lag of approximately 7-9 hours before the cells' doubling could be recorded. The spoT1 derivative of MG1655, KP31, took 2-3 hours under the same condition (data not shown). The growth in the nutrient rich LB medium was slow as well, more so at $30^{\circ} \mathrm{C}$ than at $37^{\circ} \mathrm{C}$ (Fig.2C). The markedly slow growth of KP32 (spoT1 rumA::CAT) and KP8 (MC4100 relA ${ }^{+}$spoT1 rumA::CAT) mutant often yielded fast growing suppressors (see below).

\section{The rumA::CAT $\square$ mutant (KP32) is unable to express normal stringent response}

KP32 (spoT1 rumA::CAT) grows poorly on minimal agar plate supplemented with 3-AT. Unlike the wild type $r e l A^{+}$strain, the $\operatorname{rumA}:$ :CAT mutant, though genetically $r e l A^{+}$, grows one notch better than the relA null mutant control on amino acid starvation plate (Fig.2D), the growth is same as on minimal medium as if 'frozen'. We confirmed by DNA sequencing that the relA gene in KP32 does not contain any mutation; also replacing the spoT1 mutation with spoT $^{+}$by P1 transduction abrogates the unusual stringent response of the KP32 mutant and the spoT $T^{+}$rumA::CAT transductant (KP24) behaves normally as $r e l A^{+}$strain with respect to growth on minimal plate, as indicated earlier (Fig.2A \& B) and on minimal plate supplemented with 3-AT (Fig.2D). We present below the evidence that RelA is overexpressed in the rumA insertion mutant, KP32 which explains most of its phenotypes.

\section{Isolation of spontaneous suppressors of the slow growth phenotype of rumA::CAT}

\section{mutant}

Growth of rumA::CAT mutant was severely affected on minimal medium to the extent that this growth defect could be used in selection of suppressor mutants. Fast growing colonies were often seen on the plate within 24 hours of incubation. The suppressors of KP8 (MC4100 relA ${ }^{+}$spoT1 rumA::CAT) were invariably relA null mutants as they did not grow on amino acids starvation plates containing 3-AT (Fig.3B), and unlike KP4 (MC4100 relA ${ }^{+}$) or KP8 (MC4100 relA ${ }^{+}$spoT1 rumA::CAT), grew as fast as the parent MC4100KP (relA1) on 
minimal medium (Fig.3A). Further, we transduced relA1 mutation from MC4100KP to KP8 (MC4100 relA ${ }^{+}$spoT1 rumA::CAT) in P1 transduction using the linked cysI::Tn10kan. One out of $10,000 \mathrm{Kan}^{\mathrm{r}}$ transductants was relAl, exhibiting the characteristic fast growth on minimal medium (Fig.3A) and unable to grow on starvation plate (3-AT) (Fig.3B). Presence of relA1 (relA::IS2) and rumA::CAT mutations was confirmed by PCR (data not shown).

Surprisingly, the fast growing variants of KP32 (MG1655 rumA::CAT spoT1) could not be tested for their relA status, as relA1 spoT1 mutant of MG1655 (KP53) was resistant to 3-AT, whereas MG1655 relA1 mutant (KP52) was sensitive (Fig.S2A). The residual activity of the RelA1 mutant protein is ruled out to be reason of 3-AT resistance of the KP53 mutant because the $\triangle P v u I I$ deletion of the chromosomal relA gene corresponding to the $\mathrm{N}$-terminal (p)ppGpp synthetic portion (119-455 amino acids) tagged to the insertion of the CAT cassette in the relA gene at the unique SalI site at position 1619 in the mutant KP54 yielded the same phenotype as relA1 spoTl mutant (Fig.S2A). This phenotype is reminiscent of suppression of the sensitivity of relA strains of Salmonella typhimurium to 3-AT by spoTI mutation (Tedin \& Norel, 2001). Although the $\square$ fast-growing mutants of KP31 (MG1655 $\square$ spoT1) $\square$ could not be directly tested for the $\square$ RelA $^{-\square}$ phenotype on 3-AT agar plate, $\quad$ pTE18 $\square s p o T^{+} \square$ transformants $\square$ of $\quad$ two $\quad$ of $\quad$ the $\square$ fast-growing $\square$ mutants $\quad$ were indeed $\square$ RelA`$^{-}$(sensitive to 3-AT, data not shown).

The result that the slow growth of KP8 (MC4100 $\square$ relA $A^{+}$spoT1 rumA::CAT) mutant is suppressed by inactivating mutation in $\square$ relA $\square$ suggests that the growth defect of the strain $\square$ is $\square$ presumably $\square$ due to oversynthesis of RelA. We surmised that the level of RelA protein is increased in the rumA::CAT which we confirmed in the experiment described below.

\section{Measurement of $\square$ RelA $\square$ protein by Western blot analysis $\square$}


We measured RelA protein amounts in different strains grown in both nutrient rich (LB) and minimal medium supplemented with glucose by Western Blot using anti-RelA antibody. From the results of densitometric analysis of more than three independent blots, it is evident that amount of RelA protein is 7-10 fold more in rumA mutant than in other strains (Fig.3C). Importantly, we found RelA protein to be increased to similar extent in rumA::CAT mutant of both MC4100 (relA ${ }^{+}$) and MG1655 background under all conditions of growth (Fig.3C). Notwithstanding this increased amounts, as indicated earlier, the reason for the lack of growth phenotype in KP24 (MG1655 rumA::CAT) is the presence of wild type $s p o T^{+}$gene. The increase in the RelA protein in the rumA::CAT mutant and not in rumA::TAC strain (KP59, KP60) is possibly due to increased transcription from a promoter-like sequence at the junction between rumA and CAT cassette sequence. We therefore estimated relA mRNA levels by semiquantitative RT-PCR.

\section{Amount of $\square$ relA transcript $\square$ is enhanced in the $\square$ rumA ::CAT $\square$ mutant $\square$}

The relA mRNA levels were elevated by $\sim 10$ fold when quantitated by comparative transcriptome analysis of MG1655, KP31 (spoT1) and KP32 (spoT1 rumA::CAT) strains (RPKM - 5:8:67 respectively) and validated by semiquantitative Reverse Transcriptase PCR in the strains grown in LB and in MB. We noted that (i) the relA transcript abundance is more in cells grown in $\mathrm{MB}$ than in LB. (ii) the transcript level was increased in rumA::CAT mutant (KP32, KP24) when compared to that in other strains (Fig.3D). Thus, we believe that the insertion of CAT cassette in rumA in cis increases the transcription of the relA gene.

Cloned $\operatorname{spoT}^{+} \square$ gene as a multicopy suppressor of the growth defect of KP32 (spoT1 $\operatorname{rum} A:: C A T)$

Genomic library of $\square$ E. coli $\square$ MG1655 was prepared in pBR322 vector and transformed into $\square$ KP32 (MG1655 $\square$ spoT1 rumA::CAT) mutant. From this library we selected 3 larger size colonies. The DNA prepared from each could complement the slow growth defect 
of $\square$ rumA::CAT $\square$ mutant, KP8 (MC4100 $\square$ relA ${ }^{+}$spoT1 rumA::CAT) and KP32 (MG1655 spoT1 rumA::CAT) (Fig. 2D) and also returned the normal stringent response of KP8 (Fig.S1A \&SB). Nucleotide sequencing revealed that the DNA in each clone contained spoT gene. Subcloned spoT $T^{+}$gene was sufficient to complement the growth defect of rumA mutant (data not shown).

\section{Measurement of $\square$ basal- and amino acid starvation induced $\square$ (p)ppGpp $\square$ levels in the}

\section{$\operatorname{rum} A$ mutant $\square$}

Intracellular levels of (p)ppGpp were measured in the cells of MG1655, KP31 (spoT1) and KP32 (spoT1 rumA::CAT) grown in the MOPS minimal medium and also with valine supplementation to cause leucine/isoleucine starvation by the method as described in (Cashel, 1994, Fernández-Coll \& Cashel, 2019). The result that there was no demonstrable difference in the (p)ppGpp levels between KP31 (spoT1) and KP32 (spoT1 rumA::CAT) strains was highly reproducible and consistent (Fig.4A, B). This is surprising and unexpected in the context that the relA gene cloned downstream of lacUV5 (trc) promoter in the plasmid pALS10/pSM10 synthesizes (p)ppGpp in response to induction of its expression by IPTG (Schreiber et al., 1991, Svitil et al., 1993) in the absence of starvation for any amino acid. The increase in the (p)ppGpp levels inhibits growth with an approximate linear relationship. The slow growth of the KP32/KP8 (spoT1 rumA::CAT) mutant was similarly expected to be due to the overexpression of relA causing elevated synthesis of (p)ppGpp, compounded by a further enhancement of the intracellular amounts due to its reduced turnover by hydrolysis-defective spoTl mutation. Also, there is very little difference, if any, in the levels of (p)ppGpp in different mutants in response to starvation (Fig.4C). The result is important in indicating that there is no defect in the amino acid starvation response in terms of the amount of (p)ppGpp synthesized under starvation condition. Thus, the inadequate growth of the KP32 (spoT1 rumA::CAT) mutant on 3-AT plate, in spite of being relA ${ }^{+}$, is due 
to the overexpression of RelA protein and not due to the slow decay of (p)ppGpp because of the spoT1 mutation, as growth of KP31 mutant on 3-AT plate is same as that of MG1655 (Fig.2C). Our observation is supported by similar results reported by (Sanchez-Vazquez et $a l ., 2019)$ which also matches the inference of the results described below on growth phenotype of transformants of RelA-CTD (see below).

\section{Low to moderate level expression of the plasmid-borne $\square$ relA $\square$ gene in the $\square$ spoT1 $\square$ mutant recapitulates the inhibitory growth phenotype of $\square \operatorname{rum} A:: C A T \square$ mutant}

We performed the experiment $\square$ with relA gene cloned in two types of plasmid vectors. (i) The $\square$ relA $\square$ gene in pGB2-relA (a kind gift from M. Cashel) $\square \quad$ is $\quad$ expressed from $\square$ lac $\square$ promoter in the low copy pSC101 replicon, pGB2 (Churchward et al., 1984). The expression of the $\square \operatorname{RelA} \square$ from $\square$ this plasmid was found to be partially $\square$ de-repressed $\square$ in MG1655 and its $\square$ spoT1 $\square$ mutant (KP31) (Fig.5A(III)), although not to a level as high as in $\square \mathrm{KP} 32$ (spoT1 rumA::CAT) $\square$ mutant. $\square$ Nevertheless, the growth phenotype of the pGB2relA transformants of spoT1 strain (KP31) was identical to that of KP32; severely inhibited in $\quad$ minimal glucose $\square$ medium $\square$ even $\square$ in $\quad$ the $\quad$ absence $\square$ of $\square \quad$ IPTG $\square$ supplementation (Fig.5A(Ia, $\square \mathrm{Ib})$ ). $\square \quad$ Similarly, the unusual stringent response of KP31 (spoT1)/pGB2 $\square$ relA $\square$ is indistinguishable from that of $\square$ the KP32 mutant (Fig. 5A (Ic)), exhibiting strikingly retarded growth on minimal agar plate supplemented with 3-AT even in the absence of $\square$ IPTG. $\square$ Importantly, levels of $\square$ (p)ppGpp $\square$ did not differ detectably between transformants of KP31 (spoT1) $\square / \mathrm{pGB} 2 \square$ and KP31/pGB2-relA $\square($ Fig.5A (IIa, IIb)), even though the $\square$ RelA $\square$ protein is elevated in the latter, and the levels were same as in $\square \mathrm{KP} 31$ (spoT1) and $\square \mathrm{KP} 32$ (spoT1 $\square$ rumA::CAT) $\square$ mutant (Fig.5A(III)). 
(ii) The relA $\square$ gene in the medium copy plasmid $\square$ pTE6 (pBAD18 $\square \mathrm{KAN}$ relA) is expressed from its $\quad$ own $\square$ promoters' $\square$ present $\quad \sim 800 \square \mathrm{bp} \square$ in $\quad$ the $\quad$ upstream $\quad$ DNA of $\square$ rumA $\square$ gene $\square$ (Fig.1) (Brown et al., 2014, Metzger et al., 1988, Nakagawa et al., 2006). Here too, expectedly, the growth of pTE6 $\square$ transformants $\square$ of $\square \mathrm{KP} 31$ (spoT1) $\square$ was drastically impaired in relation to those of MG1655, more acutely in minimal medium (Fig.5B (Ia, Ib)) than in LB (data not shown). Furthermore, the stringent response of the $\square$ spoT1 $\square$ transformants $\square$ is $\quad$ abnormal, similar $\quad$ to $\quad$ that $\quad$ of $\square \mathrm{KP} 32 \quad$ (spoT1 rumA::CAT) $\square$ mutant, $\square$ in that $\square$ the poor growth on amino acid starvation plate (minimal agar plate supplemented with 3-AT) is $\square$ almost $\square$ same as that on minimal agar (Fig.5B (Ic)). $\square$ The result clearly indicates that the phenotype of $\square \mathrm{KP} 32$ (spoT1 rumA::CAT) could be also reproduced by $\square$ moderate level expression of $\square$ relA $\square$ gene using the second plasmid system, although the possibility of levels of (p)ppGpp being higher in the spoT1 mutant which may reduce the growth, cannot be ruled out.

(iii) The plasmid pALS10 (a gift from M. Cashel) extensively used for ectopic production of (p)ppGpp expresses the $\square$ RelA $\square$ protein under the control of the $\square$ tac $\square$ promoter in $\square$ colEl $\square$ replicon $\square$ (Schreiber et al., 1991). The growth phenotype of the transformants of spoT1 mutant merits attention (Fig.5C). The colonies $\square$ were small, sick and extremely slow growing on LA agar in the absence of induction of $\square$ RelA $\square$ by IPTG, and fail to regrow. The plasmid also affects the growth of MG1655 strain $\square$ appreciably in comparison to $\square$ pALS14 (containing the inactive $\square$ relA $\square$ gene) $\square$ (Fig.5C), $\square$ the phenotype described for long in the literature (Schreiber et al., 1991, Svitil et al., 1993). $\square$ We believe that the retardation of the growth of MG1655 by multiple copies of $\square$ relA $\square$ is independent $\square$ of $\square$ (p)ppGpp $\square$ synthesis, at least partially, for following reasons - (i) pALS10 plasmid contains the gene for $\square$ lacI $\square$ repressor for increasing the host range of 
the $\square$ plasmid $\square$ and $\square$ for regulated expression of $\square$ relA $\square$ so that the basal level of expression of the $\square$ relA $\square$ is not toxic in multiple copies. Indeed, the $\square$ (p)ppGpp $\square$ levels in the cells of MG1655 carrying pALS10 or its equivalent (Mechold et al., 1996, Schreiber et al., 1991, Svitil et al., 1993, Zhu \& Dai, 2019) grown in the medium lacking IPTG is comparable to the cells containing pALS14 even $\square$ though the $\square$ un-induced $\square$ levels of $\square$ RelA $\square$ protein are significantly high (Schreiber et al., 1991). This observation is the basis of the authors' (Sanchez-Vazquez et al., 2019) surmising that the cellular pool size of (p)ppGpp is dictated by the relA/spoT genotype of the host and is independent of the basal level expression of the cloned copy number of relA. (ii) $\square$ A recent finding by $\square$ Turnbull et al., (2019) $\square$ demonstrates a strong inhibition of the growth by high levels expression of the C-terminal fragment of $\square$ RelA $\square$ supporting $\square$ the contention that the inhibitory effects of $\square$ RelA $\square$ overexpression $\square$ may involve $\square$ (p)ppGpp-independent component as well. $\square$ The steady state pool size $\square$ of $\square$ (p)ppGpp $\square$ in KP31 (spoT1)/pALS10 $\square$ cannot $\square$ be estimated due the $\square$ lethality of the plasmid.

Overexpression of fusion of N-terminal (1-119 amino acids) with RelA-CTD (454-744 and 406-744) renders growth inhibition by RelA-CTD, (p)ppGpp dependent.

Our observation that the increased level of expression of RelA in rumA::CAT spoT1 mutant (Fig.3 C) or the leaky expression of plasmid copies of relA in the absence of the inducer IPTG (Fig.5III) is not associated with a corresponding increase in the (p)ppGpp levels (Fig4; Fig.5A (IIa, IIb)), is not without precedence (Sanchez-Vazquez et al., 2019). This observation applied to both wild type (Schreiber et al., 1991, Gropp et al., 2001) and to the spoT1 mutant (Fig 4; Fig.5A (IIa, IIb)), albeit the level of (p)ppGpp is characteristically higher in the spoT1 mutant than in the wild type and independent of rumA::CAT mutation in KP32 or presence of additional copies of plasmid encoded relA in KP31/pGB2 relA (Fig.4II, Fig.5 (IIb)). Strikingly, the growth retardation of the spoT1 mutant containing multiple copies of RelA is 
more severe than that of the spoTl mutant itself, even though the levels of (p)ppGpp are the same in each of the two conditions (Fig. 2A, 2B, 2C, 5A (Ia, Ib), 5B (Ia, Ib), 5C).

The result led us to assume that the RelA's (p)ppGpp synthesis function may not be involved in the spoT1 mutation-dependent growth retardation phenotype. It is thus reasonable to test the growth effects of RelA-CTD in the spoTl mutant. As we understand, the N-terminal domain (1-390) of the RelA protein consists of 1-200 amino acids constituting inactive hydrolytic (HD) domain and 201-390 amino acids comprising $\square$ (p)ppGpp $\square$ synthesis domain (SYN). $\square \square$ Thus, presuming the N-terminal 119 amino acids to be devoid of any discernible function, the simple strategy of cloning CTD involved removing sequences internal to the two $\square P v u I I \square$ sites at positions 354 and 1362 in the $\square$ relA $\square$ gene. This fortuitous cloning uncovered an unexpected layer of regulation of $\square$ relA.

Two types of in-frame fusion of N-Terminal 119 amino acids with RelA-CTD were constructed starting with the plasmid pTE6 (pBAD18 $\square$ Kan relA). (i) The plasmid construct pTE22 represents the $\mathrm{N}$-terminal 119 amino acids fused to the $\mathrm{C}$-terminal domain comprising of $\square 455-744 \square$ amino acids, generated by $\square P v u I I \square$ digestion and intramolecular ligation $\square$ of the $\square$ relA $\square$ DNA in the plasmid $\square$ pTE6 $\square$ (pBAD18 $\square$ KANrelA). The $\square$ truncated $\square$ RelA $\square$ is insufficient in complementing the $\square$ stringent response $\operatorname{defect} \square$ of $\square$ relAl $\square$ mutation in MC4100 (Fig.S2 B). The fact that substitution of G251E $\square$ by site-directed mutagenesis results in inactive mutant protein that has lost $\square$ (p)ppGpp $\square$ synthesis ability (Gropp et al., 2001), and that the $\square P v u \mathrm{III} \square$ deletion removes the critical G251 $\square$ amino acid important for $\square$ (p)ppGpp $\square$ synthesis, reaffirms the functional $\square$ inability $\square$ of the truncated protein to synthesise $\square$ (p)ppGpp.

(ii) The plasmid construct pTE23 represents in-frame fusion of N-terminal 119 amino acids with the 406-744 portion of RelA-CTD (Turnbull et al., 2019), generated by PCR (described in Materials and Methods). An additional, intact TGS domain (200-396) in pTE23 
however, is not required for binding to the ribosomes (Gropp et al., 2001, Turnbull et al., 2019).

Remarkably, the growth of the transformants of spoT1 mutant with each of the two plasmids (pTE22, pTE23) was indistinguishable; retarded to the same $\square$ extent $\square$ on both minimal agar containing glucose as the sole carbon source and on minimal medium supplemented with 3-AT but less than that by the full length $\square$ relA (pTE6) whereas $\square$ transformants $\square$ of $\square$ MG1655 were unaffected for growth (Fig.5B (Ia, Ib, Ic)). The severity of the effect on growth in minimal medium is evidently due to $\square$ (p)ppGpp $\square$ levels being high in minimal glucose medium (nutrient poor) $\square$ than in LB.

\section{Abrogation $\square$ of the reduced growth effect of $\square$ moderate expression of $\square$ RelA-CTD by $\square$ relA1 $\square$ mutation is reversed by $\square \operatorname{spoT202} \square$ mutation $\square$}

The result that growth of the spoTl mutant is retarded by moderate level expression of RelACTD in two fusion constructs, pTE22 (1-119 NTD fused to 406-744 CTD) and pTE23 (1-119 NTD fused to 454-744 CTD), which is without any effect in the wild type strain (Fig. 5B (Ia, Ib)) suggests that the higher basal levels of (p)ppGpp in the spoT1 mutant may be required for manifestation of the growth effects. In agreement with this assumption, the relAl mutation, which lowers (p)ppGpp levels in the spoT1 mutant, also abolishes the spoT1specific effects of growth by the RelA-CTD (Fig.6 (Ia, Ib)). If the growth effects of RelACTD are indeed accentuated by higher basal levels of intracellular levels of (p)ppGpp, it should be possible to restore slow growth phenotype of the RelA-CTD in spoT mutants that contain levels of (p)ppGpp higher than in the relAl spoTl mutant. Mutant $\square$ spoT $\square$ alleles, $\square$ spoT202, $-203, \quad-204 \square$ that are increasingly compromised for their $\square$ (p)ppGpp $\square$ hydrolysis function $\square$ have been described to reverse the 3-AT sensitive phenotype of $\square$ relAl $\square$ mutants as a result of the enhanced (p)ppGpp levels (Sarubbi et al., 1988),Fig.S4 B), and accordingly exhibit slow growth (Fig.S4 A). Because of the increasing 
severity of the $\square$ spoT $\square$ alleles on growth, $\square$ we $\square$ chose to work with $\square$ spoT202 $\square$ mutation, which is more severe than $\square$ spoTl $\square$ in its ability to elevate $\square$ (p)ppGpp $\square$ levels $\square$ but less than $\square$ that $\quad$ by $\square$ spoT203/204 $\square$ (Sarubbi et al., 1988), KP56 (relAl

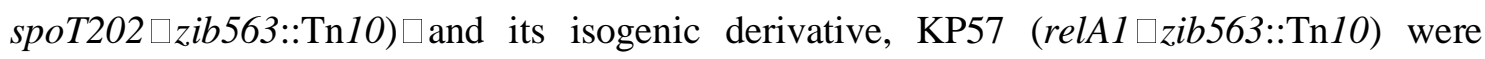
constructed $\square$ by $\quad$ P1-mediated $\square$ cotransduction $\square$ of $\square$ spoT202 $\square$ mutation with $\square$ zib563::Tn10 $\square($ Table 1). $\square \square$ The control vector, pBAD18Kan, $\square$ pTE22 (RelACTD1) $\square$ and pTE23 (RelA-CTD2) were each transformed in the isogenic pair and the growth was measured qualitatively (Fig.6II A) $\square$ and quantitatively (Fig.6II B). $\square$ The $\square$ phenotype of effect $\square$ of overexpression of $\square$ fusion RelA-CTD $\square$ on $\square$ growth $\square$ was largely reproduced in the spoT202 mutant, $\quad$ in $\quad$ that $\quad$ the $\square$ pTE22 $\square$ (RelA-CTD1) $\square \square$ and pTE23 (RelACTD2) transformants $\square$ of $\square$ KP56 (relA1 spoT202) were $\square$ appreciably $\square$ slow $\square$ growing $\square$ in comparison to $\square$ the control vector $\square \mathrm{pBAD} 18 \mathrm{Kan} \square$ transformants $\square$ (Fig 6 (IIa, IIb)), strongly indicative of the regulation of C-terminal function by $\square$ (p)ppGpp. $\square \square$ The longer lag in the growth of KP31/ (pTE22/pTE23) $\square$ transformants $\square$ (Fig. 5B (Ib)) $\square$ in comparison to that of KP56 /pTE22 (Fig.6II B) might be due to $\square$ the $\square$ potential for dynamic changes in the concentration of $\square$ (p)ppGpp $\square$ effected by $\square$ RelA $\square$ in the $\square$ nutrient poor minimal medium in the former strain than in the latter. $\square$ Furthermore, the growth of KP58 (spoT202 $\square$ relA $A^{+} \square$ ) $\square \square$ transformants $\square$ carrying $\quad$ pTE22 $\quad$ or $\quad$ pTE23 $\square \quad$ was $\square$ severely reduced $\square$ in comparison to that of pBAD18kan $\square$ plasmid $\square$ transformants $\square \square$ (Fig.6C (I, II)). It must be noted here that, unlike the observation of $\square$ (Sarubbi et al., 1988) $\square$ which describes an inability of $\square \operatorname{spoT} \square 202 / 203 \square$ mutation to coexist with $\square$ relA $A^{+}$, we were able to transduce $\square$ each of the $\square$ spoT $\square$ mutation $\square$ into $\square$ the wild type MG1655 strain. As expected, growth of $\square$ relA $A^{+} \square$ spoT $\square 202 \square$ transductants, KP58, $\square$ was $\square$ drastically $\square$ slow when compared to that $\quad$ of $\square$ relA1 $\quad$ spoT202 $\square$ mutants $\square$ (Fig.S4A). $\square \square$ Growth $\quad$ of $\quad$ KP56 $\square \quad$ (relA1 spoT202)/pTE22 (RelA-CTD1) $\square$ transformants $\square$ on minimal agar plate supplemented with 3- 
AT was similarly compromised/reduced to the same extent as on minimal agar (data not shown). These results convincingly demonstrate that the growth effects of $\square$ multicopy $\square$ expression of $\square \operatorname{RelA}-\mathrm{CTD} \square$ are $\square$ indeed potentiated by higher levels of intracellular $\square$ (p)ppGpp $\square$ and $\square$ that the enhanced levels of $\square$ (p)ppGpp $\square$ effected either by spoT1 $\square$ or $\square$ relA $1^{\square}$ spoT202 $\square$ mutations are sufficient for the manifestation of the growth retardation/abnormal stringent response $\square$ phenotypes. $\square$ Given the known function $\square$ of the Cterminal domain of $\square \operatorname{RelA} \square \square$ to bind ribosomes $\square \square($ Gropp et al., 2001), $\square$ our results seem to suggest that this binding is modulated by the $\square$ levels of $\square$ (p)ppGpp in the cell; $\square$ and since $\square$ RelA-CTD binding to ribosomes has been shown to slow translation rates (Turnbull et $a l ., 2019)$ the effect on the growth can be rationalized.

In summary, the following results likely contribute to further our understanding of the regulation of $\square \operatorname{RelA} \square$ and its (p)ppGpp $\square$ synthesis function. Sustained low to moderate level of expression of $\square \operatorname{RelA} \square$ in $\square$ the spoT1 mutant was associated with phenotypic consequence of slow growth, clearly absent in the wild type strain, suggesting that higher than the normal basal level of $\square$ (p)ppGpp $\square$ is required for the manifestation of the growth phenotype. $\square$ This is further validated by reproduction of a large part of the growth retardation effect by expression of RelA-CTD (Fig. 5B (Ia, Ib)), and abolition of the slow growth phenotype by decreasing the intracellular levels of (p)ppGpp in the spoTl mutant by relAl mutation (Fig.6A (Ia, Ib)). The requirement of higher levels of (p)ppGpp for RelA-CTD mediated inhibition of growth can be also met by substitution of $\square$ chromosomal RelA's $\square$ (p)ppGpp $\square$ synthesis function with spoT202 mutation which elevates intracellular $\square$ (p)ppGpp $\square$ levels in $\square$ relAl $\square$ mutant due to the an inactive hydrolytic activity of $\square$ the SpoT $\square$ protein $\square$ (Fig.6 (IIa, IIb))(Sarubbi et al., 1988). We were able to assign this (p)ppGpp regulatory function to the 119 amino acids NTD domain as its in-frame fusion with RelA-CTD, both 406-744 (RelA-CTD2) and 455-744 (RelA-CTD1) rendered the growth inhibitory effects of its expression dependent on the 
elevated cellular levels of (p)ppGpp. Our findings may $\square$ suggest a mechanism that provides a rationale for in vivo regulation of $\square \operatorname{RelA}$ ’s $\square$ (p)ppGpp $\square$ synthesis activity.

\section{Discussion:}

Our results seem to unfold a new aspect of the regulation of RelA demonstrating that the aberrant stoichiometry of RelA (more precisely RelA-CTD) to ribosomes is inhibitory to growth, the severity of which is determined by an incremental rise in the basal levels of (p)ppGpp, therefore independent of its synthesis by RelA. This inference is apparent from our unanticipated result that mutational and/or growth condition mediated elevation of (p)ppGpp pool size potentiates inhibition of growth by moderate level expression of RelACTD. Expression of in-frame fusion of the N-terminal 119 amino acids of the HD domain with the RelA-CTD (406-744, containing TGS domain) or (454-744, lacking the TGS domain) was equally effective in causing growth inhibition of spoT1 mutant, but not that of wild type. Our results are compatible with the model that the growth inhibitory effect of RelA-CTD is due to its interaction with the target ribosomes which is modulated by (p)ppGpp levels in the cell, the toxicity effects, are nevertheless independent of the (p)ppGpp synthesis function of RelA.

It must be noted that, unlike the growth inhibitory effect of high-level expression of RelACTD, the growth phenotypes we describe here are more pertinent and relevant to physiological concentration of RelA because the protein is expressed from its own promoters in a low copy to medium copy vector and translated from its own ribosome binding site (SD sequence) not optimised for very high-level expression (Turnbull et al., 2019). Importantly, the high-level multicopy expression of RelA-CTD ${ }^{454-744}$ (a gift from M Roghanian) inhibits growth of strains irrespective of their genotype; on the contrary, expression of the same 
RelA-CTD gene in a low copy plasmid, pNDM220:relA ${ }^{\mathrm{CTD}}$ (a gift from M Roghanian) does not affect either the growth of wild type or its spoTl derivative (data not shown) or interfere with the expression of stringent response (Turnbull et al., 2019), proving that NTD's HD domain contains features for (p)ppGpp-dependent regulation of growth effects of RelACTD.

Some of the observations pertinent to our studies regarding the ectopic expression of plasmid-borne relA from a controlled promoter is the lack of proportional increase in the (p)ppGpp levels and the extent of growth inhibition induced by overexpression of full length RelA (Schreiber et al., 1991, Gropp et al., 2001), explained earlier as being due to toxicity of full length RelA protein (Schreiber et al., 1991). The implication of this and the results presented here is that the growth inhibitory effects of RelA are due to (i) the product, (p)ppGpp-mediated inhibition of transcription of stable RNA and ribosomal protein synthesis and (ii) (p)ppGpp-responsive altered binding of RelA-CTD to ribosomes, slowing rate of protein synthesis. In the recent study, Sanchez-Vazquez et al., (2019) concluded that the leaky basal level expression of active RelA does not alter the (p)ppGpp levels in the cells which are rather dictated by the chromosomal status of relA/ spoT genes, which strikingly corroborate ours (Fig. 4, Fig 5A (IIa, IIb)). Our finding that growth inhibition effect of leaky, un-induced, low (Fig. 5A (Ia, Ib)) to moderate level expression of RelA is more acute in the spoT1 mutant than in the wild type strain and that the un-induced multiple copy expression is lethal in the spoT1 mutant (Fig.5C), merits investigation.

How relevant is our observation? The positive activation of RelA by its own product, (p)ppGpp (Shyp et al., 2012), explains the rapid increase in the concentration of (p)ppGpp in the cell in response to the signal of amino acid starvation, mandating a negative component of regulation of its synthesis as well. When the concentration 
of (p)ppGpp reach the threshold required for a global effect on transcriptome and proteome, its cascading effect must be countered/tempered by a negative regulation of RelA's synthesis activity. One of the proposed negative means of regulation of RelA activity is a decrease in the (p)ppGpp levels affected by restoration of the amino acid pool under favourable growth condition. It makes sense that the mechanism proposed here - the RelA-CTD interaction with the target ribosome is modulated by incremental rise in (p)ppGpp which possibly inhibits its synthesis by the $\mathrm{N}$-terminal, complements the negative regulation. This continuous responsiveness of RelA function to (p)ppGpp levels in the cell could likely be an in vivo mechanism of negative modulation of ( $\mathrm{p}) \mathrm{ppGpp}$ synthesis. The in vitro observation that (p)ppGpp synthesis is maximum at less than 10:1 of 70S:RelA ratio and the synthesis decreases as the stoichiometry increases (Wendrich et al., 2002, Kudrin et al., 2018) seems to support our finding that moderate level of RelA overexpression does not lead to (p)ppGpp synthesis in vivo (Schreiber et al., 1991, Gropp et al., 2001).

The possibility is worth considering that the increase in the levels of RelA in the exponentially growing cells slow the growth by affecting the higher affinity of EFTu.tRNA.GTP ternary complex at the A site on the ribosome (Winther et al., 2018, Loveland et al., 2016).

Interestingly, results of Syal et al., (2015) point to similar conclusions. They showed that Rel-CTD of M. smegmatis binds to (p)ppGpp and suggest this binding to be important for providing a negative feedback mechanism of regulation to adjust the (p)ppGpp levels in the cell. They confirmed that mutations in the Rel protein implicated in the binding of (p)ppGpp accordingly increased $\mathrm{p}(\mathrm{p}) \mathrm{ppGpp}$ synthesis and reduced hydrolytic activity.

This work raises the question - has RelA's catalytically inactive HD got any role or function for the protein? The evidence is largely tantalizing. On the lines that there is reciprocal inter- 
domain regulation between HD and the SYN domains of the NTD of long RSHs (Tamman et al., 2019, Takada et al., 2021), it is interesting to note that mutations in the inactive HD domain of E .coli RelA reducing the SYN activity (Montero et al., 2014, Spira \& Ospino, 2020) may reflect the relic of this regulation. Although the catalytic amino acid residues of the HD domain are clearly absent in the monofunctional RelA, there is evolutionary conservation of the HD sequence all along this region between bifunctional RSHs and RelA, suggesting a possible important function. Recently, Takada et al., (2021) have clearly demonstrated the localization of (p)ppGpp binding site in the NTD domain of RelA protein not involving the catalytic SYN site. In light of this, our result that the (p)ppGpp mediated regulatory function is present in the NTD's HD domain for regulating the growth inhibitory function of RelA-CTD may not seem implausible.

\section{Materials and Methods:}

\section{Growths Conditions: $\square$}

Bacterial cells were normally grown in Luria Bertani (LB) broth or in M9 minimal broth (MB) at $37^{\circ} \mathrm{C}$ or $30^{\circ} \mathrm{C}$ with shaking. $2 \times Y$ T broth was used for bacterial cell growth during electroporation. When necessary, media were supplemented with Kanamycin $(50 \mu \mathrm{g} / \mathrm{ml})$, Ampicillin $(100 \mu \mathrm{g} / \mathrm{ml})$, Chloramphenicol $(12 \mu \mathrm{g} / \mathrm{ml})$, Tetracycline $(10 \mu \mathrm{g} / \mathrm{ml})$, Streptomycin $(100 \mu \mathrm{g} / \mathrm{ml})$, Spectinomycin $(50 \mu \mathrm{g} / \mathrm{ml})$. Isopropyl $\beta$-D-thiogalactopyranoside(IPTG) was used at a final concentration of $1 \mathrm{mM}$. DNA manipulations were carried out according to protocols described in (Sambrook, 1989). P1 transduction was carried out by method described by (Miller, 1992). Strains used in this study are the derivatives of $\square$ E. coli $\square$ K-12. Strains and plasmids are listed in Table 1. Primers used in this study are listed in Table 2. $\square$

\section{Methods:}

\section{Insertion of $\square C A T / K A N \square$ cassette in chromosomal $\square$ rumA $\square$ gene $\square$}


The rumA amplicon (1529 bp), generated using RumAF1-RumARKpnI primers, was cloned in to the broad host range vector pBBR1MCS2 (Kovach et al., 1995) and vector pBluescriptKS at EcoRI-KpnI sites yielding pTE14 and pTE19 respectively.

1. Amplification of $824 \square$ bp $C A T \square$ gene from pACYC184 vector (Bartolome et al., 1991) was carried out by 2 pairs of CAT primers. (i) $\square$ CATFSalI $\square$ and $\square$ CATRSalI $\square$ primers were used for cloning $\square C A T \square$ amplicon into pTE14 vector at unique $\square$ SalI $\square$ site to generate pTE15, (ii) Using CATFMB and CATRMB primer pair, $\square C A T \square$ amplicon was inserted at $\square M l u \mathrm{I} \square$ site in $\square$ rumA to $\square$ generate pTE16 construct. $\square$

2. Amplification of $944 \square$ bp $K A N \square$ gene from pBAD18Kan Vector (Guzman et al., 1995) was carried out by KAN primers $\square$ KANLeftSalI $\square$ and $\square$ KANRightSalI $\square$ and cloned at unique $\square$ SalI $\square$ site of pTE19 to generate pTE20. Insertion of $\square C A T / K A N \square$ cassette at the plasmid level was confirmed by restriction digestion. $\square$

We replaced chromosomal $\square$ rumA $\square$ gene with the mutant allele by $\square$ recombineering $\operatorname{in} \square E$. coli $\square$ strain DY330 (Yu et al., 2000) and also using the portable pKD46 plasmid (Datsenko \& Wanner, 2000) in MG1655 strain. The linear PCR DNA of mutant rumA gene containing CAT (2335 bp) or KAN cassette (2455 bp) was amplified using RumAF1-RumARKpnI primers and the plasmids pTE15 and pTE20 used as templates. The PCR DNAs were purified by gel elution method and transformed into electrocompetent cells. The genomic mutations in rumA containing insertion of $C A T / K A N$ in each case were transduced into a fresh background of MC4100 and MG1655 through P1kc-mediated generalized transduction as described by (Miller, 1992). The orientation of antibiotic cassette was determined by PCR using antibiotic cassette specific forward primer in combination with rumA gene specific forward and reverse primers (data not shown). In the transduced MC4100 derivatives, the $\mathrm{relA}^{+}$gene was also confirmed by PCR and sequencing (data not shown). Similarly, the P1 transduction- 
mediated transfer of spoT1 mutation in MG1655 was confirmed by DNA sequencing of the representative strains.

\section{Construction of $\square \mathrm{KP4}\left(\mathrm{MC4100} \square \mathrm{relA}^{+}\right)$}

The strains used in the study were constructed by P1 transduction. $\square$ relA $A^{+} \square$ allele was $\square$ cotransduced $\square$ into $\quad$ MC4100KP $\quad$ (relA1 spoT1) with $\square$ cysI3152::Tn10kan $\square(\mathrm{CAG} 12182)$. For markerless strain construction in this cross, CysI $I^{-}$transductants were converted into $\mathrm{CysI}^{+}$prototrophs, again by $\mathrm{P} 1$ transduction, screening for loss of $\square \operatorname{Kan}^{\mathrm{r}} \square$ gene. The $\square$ relA $\square$ gene structure was confirmed by PCR using $\square$ RelAF $\square-\square$ RelAR $\quad$ primers. $\square \quad$ relAl $\square$ gene of $\quad$ MC4100 generates $3.561 \mathrm{~Kb} \square$ amplicon $\square$ due to an IS2 $(1.327 \mathrm{~Kb})$ insertion in the $\square$ relA $\square$ whereas the size of the wild type $\square$ relA $\square$ gene is $2.234 \mathrm{~Kb}$ (data not shown). $\square$

\section{Exchange of $\square$ spoT $\square$ alleles from MC4100 to MG1655 $\square \square$}

The spoT1 allele of MC4100KP was first linked to $\square \Delta p y r E 748:: K A N \square$ (KP11) $\square$ marker ( $67 \% \square$ cotransduction), followed by its transfer into MG1655 and KP24 through P1 transduction generating KP29 (spoT1 $\square$ ApyrE748::KAN) and KP30 (rumASalI::CAT spoT1 $\square \Delta p y r E 748:: K A N)$ respectively; this is followed by selection of $\square p y r E^{+} \square$ derivatives, KP31 (spoT1) and KP32 (spoT1 rumASalI::CAT) on minimal medium in the absence of uracil.

\section{Assessment of $\square$ relA $\square$ phenotypes $\square$}

The growth phenotypes of $\mathrm{RelA}^{+}$and $\mathrm{RelA}^{-}$were scored on one of the three types of plate as described below. (i) M9 minimal medium supplemented with glucose $(0.2 \%)$ and all amino acids except histidine $(4 \mu \mathrm{g} / \mathrm{ml})$, adenine $(1 \mathrm{mM})$, thiamine $(1 \mathrm{mM})$, and 3-AT $(15 \mathrm{mM})($ Silva \& Benitez, 2006). (ii) M9 glucose medium containing serine, methionine, glycine, (SMG) (100 $\mu \mathrm{g} / \mathrm{ml}$ each), adenine $(50 \mu \mathrm{g} / \mathrm{ml})$, thymine $(50 \mu \mathrm{g} / \mathrm{ml})$, and calcium pantothenate $(1$ $\mu \mathrm{g} / \mathrm{ml}$ ) (Silva \& Benitez, 2006). (iii) Amino acid starvation was induced by addition of 0.5 - 
$1 \mathrm{mg} / \mathrm{ml}$ serine hydroxamate in minimal medium containing $0.2 \%$ glucose (Tosa \& Pizer, 1971).

\section{Determining growth}

Growth curve experiments were performed in either LB broth and/or M9 Minimal broth. Overnight grown cultures were diluted $1: 100$ in $50 \mathrm{ml}$ fresh media, shaken at $37^{\circ} \mathrm{C}$ temperature, unless specified otherwise. $\mathrm{OD}_{600}$ was monitored. Growth rate experiments were repeated thrice.

\section{Isolation of $\operatorname{spoT}^{+}$gene in pBR322}

A MG1655 genomic DNA library, prepared by partial digestion of chromosomal DNA with Sau3AI, was cloned into the BamHI site of pBR322. P1 lysate made on the library of pool of 10,000 independent clones was transduced into KP32 (rumASalI::CAT) with selection for plasmid-encoded ampicillin resistance on minimal ampicillin agar plate. Transductants were screened for faster growing colonies. Plasmids were prepared from 3 independent colonies that permitted fast growth of KP32 on minimal medium and restored growth on minimal medium + 19 amino acids lacking His supplemented with 3-AT (normal relA ${ }^{+}$phenotype).

\section{Quantitation of RelA Protein expression by Western blot}

Cultures in mid-exponential phase at an $\mathrm{OD}_{600}$ of 0.6 . The cells were centrifuged and the pellet was resuspended in Laemmli buffer and sonicated. An estimated $20 \mu \mathrm{g}$ protein was loaded on a $10 \%$ SDS-Polyacrylamide gel and then electrotransferred to Nitrocellulose membrane (Bio-Rad). The blots were probed overnight with the monoclonal anti-RelA primary antibody (1:2000; Santa Cruz) and anti- $\beta$-Galactosidase primary antibody (1:5000; Novusbio) at $4^{\circ} \mathrm{C}$. Anti-mouse/rabbit IgG conjugated with HRP (1:2500) was used as secondary antibody. Finally, membranes were developed and visualized with Enhanced Chemiluminescence Western blotting detection system (Bio-Rad). Images were scanned for densitometric analysis using the Image $\mathbf{J}$ software. 


\section{RNA isolation and mRNA expression of relA gene by semi-quantitative RT PCR}

RNA was isolated from strain of MG1655 derivatives using the TRIzol reagent (Invitrogen).

Genomic DNA in the sample was removed with DNAseI kit (Invitrogen). A reversetranscription into first strand c-DNA reaction was performed using $2 \mu \mathrm{g}$ RNA with MuLV reverse transcriptase kit in a $20 \mu \mathrm{L}$ reaction volume (Invitrogen) as per manufacturer's instructions. cDNA was amplified using gene specific primers (Table 2). GAPDH was used as an internal control and the PCR amplicons were analyzed by electrophoresis on $2.0 \%$ agarose gel. Images were scanned for densitometric analysis using the Image $\mathrm{J}$ software.

\section{Measurement of (p)ppGpp levels by thin layer chromatography}

Estimation of (p)ppGpp was carried out as described in (Cashel, 1994) and (Fernández-Coll \& Cashel, 2019). Briefly, Growth conditions were achieved by continuously shaking 24 well plates in a Thermomixer (Eppendorf) and monitoring $\mathrm{OD}_{600}$ in unlabeled parallel cultures in the synergy HT plate reader (Biotek). Overnight cultures were grown in MOPS (3-(Nmorpholino) propanesulfonic acid) media with $3 \mathrm{mM}$ sodium phosphate and $0.2 \%$ Glucose. Cultures were then diluted into the low phosphate medium $(0.2 \mathrm{mM})$ to give specific activities sufficient for nucleotide labelling with $100 \mu \mathrm{ci}$ of $\mathrm{P}^{32}$ in $600 \mu$ lof total volume per well and grown till $\sim 0.4 \mathrm{OD}_{600}$. When necessary valine was added to induce isoleucine starvation. $20 \mu \mathrm{l}$ of samples were collected in a tube containing equal volume of $6 \mathrm{M}$ formic acid, then frozen at $-20^{\circ} \mathrm{C}$. The samples were subjected to three cycles of freeze-thaw and then centrifuged. $5 \mu 1$ of the supernatant was applied on surface of TLC PEI-cellulose (Millipore) and resolved in $1.5 \mathrm{M} \mathrm{KH} 2 \mathrm{PO} 4, \mathrm{pH} 3.4$. The chromatogram was dried at room temperature and the $\mathrm{pH}$ front portion containing $32 \mathrm{Pi}$ removed. The autoradiograph was exposed overnight on phosphor screens. Finally, the nucleotide spots were visualized by phosphoimager (Typhoon 9400 imager) and quantified with imageJ. The amounts of ppGpp 
and pppGpp were normalized to the sum of the pppGpp+ppGpp+GTP (referred as total G) observed in the same sample.

\section{Construction of in-frame fusion of RelA-CTD with 1-119 amino acids RelA-NTD:}

The in-frame fusion of 1-119 amino acids of RelA-NTD to 454-744 amino acids of RelACTD was carried out by removal of relA gene sequence between two $P v u I$ sites at positions 354 and 1362 in the pTE6 (pBAD18kan relA $^{+}$) plasmid. In-frame fusion of 1-119 amino acids of RelA-NTD to 406-744 of RelA-CTD was carried out by overlapping PCR in two steps. In the first step, two individual PCRs, one each for the NTD and the CTD, were generated. 1-357 bp PCR product for NTD containing 1-119 amino acids was obtained using the primer pair RelAL and RelAfusionprimerRgt. RelA-CTD PCR product from 1014$2235 \mathrm{bp}(406 \quad$ amino acids) was obtained using primers RelAFusionprimerLFT and RelAR. The PCR products of the NTD and CTD reactions were mixed in 1:1 proportion and used as templates for PCR amplification of the fusion product using primers RelAL and RelAR. The fusion PCR DNA (1578 bp) was used to repair the pTE22 (relA $\triangle P v u \mathrm{II})$ linearized at $P v u \mathrm{II}$ site by recombineering in host MG1655/pKD46. The insert DNA from each of the two clones (pTE23) was validated by restriction enzyme digestion and also confirmed by DNA sequencing.

Acknowledgement: Krishma Tailor received financial support from UGC, Govt. of India. Authors are thankful to $\mathrm{M}$. Cashel and M. Roghanian for their help with plasmids and strains. The authors declare no conflict of interest.

Table and Figure Legends

Table1: Strains and plasmids used in this study

Table 2: List of Primers used in this work 
Fig. 1: Genetic organization and transcriptional signals of the relA gene in the upstream rumA gene on $\boldsymbol{E}$ coli chromosome: The four arrows (P1, P2, P3, and P4) indicate the known relA promoters and the numbers represents the +1 start position of the transcript from each of the four promoter in the rumA gene. The triangle indicates $C A T$ antibiotic cassette insertion at the SalI site -878 bp upstream $\square$ of all the known promoters of $\square$ the $\operatorname{relA} \square$ gene. The arrow on CAT cassette indicates transcription direction of CAT. The AUG represents start site of relA translation.

Fig. 2: $\operatorname{rumA}:$ :CAT mutant grows poorly on both minimal medium and on amino acid starvation plates: (A) Growth of the rumA mutant on minimal agar plate at $30^{\circ} \mathrm{C}$. (B) Growth curve measurement of spoT1 rumA and spoT1 mutants of MG1655 strains in minimal broth at $37^{\circ} \mathrm{C}$ and in $(\mathbf{C}) \mathrm{LB}$ at $30^{\circ} \mathrm{C}$. Growth curve is representative of three independent experiments. (D) The growth of rumA mutant on minimal agar plate supplemented with 3AT.

Fig. 3: Moderate level expression of RelA in the context of spoT1 mutation is the cause of phenotypes of rumA::CAT mutant: Suppressors of slow growth of the rumA::CAT mutant are RelA*. The growth phenotype of fast-growing variants of KP8 was assessed on (A) minimal agar plate and (B) minimal agar plate supplemented with 3-AT. The plates were examined after 24 hours of incubation at $37^{\circ} \mathrm{C}$. (C) Quantification of RelA protein by Western blot. RelA protein was measured in cell extract of (I) rumA mutant derivatives of MC4100 grown in rich media (LB) and in minimal media (MB); (II) cell extracts of rumA mutant derivatives of MG1655 grown in $\mathrm{LB}$ and in $\mathrm{MB}$ at $37^{\circ} \mathrm{C}$. Upper panel represents genotypes of mutants of MC4100 (I) and MG1655 (II) derivatives. Fold differences in protein levels of rumA::CAT relative to wild type represents average of the three independent 
experiment and indicated against each lane. In immunoblots of MG1655 strains, the lower panel (LP) shows $\beta$-Galactosidase protein as an internal protein control detected by anti- $\beta$-gal antibody. (*) Insertion of the CAT cassette in the rumA gene (rumA::TAC) in the orientation opposite to that of $\operatorname{rumA}:: C A T$. (D) Semi-quantitative measurement of relA transcript by Reverse Transcriptase-PCR. The cells were harvested for isolation of RNA after their growth in rich media (LB) and minimal media (MB). Upper panel shows 426 bp relA amplicon generated using RelA RT primers (Table 2) corresponding to C-terminal portion. Lower panel represents RT-PCR of internal control of gapdh mRNA. 100 bp ladder was used as M.W marker.

Fig. 4: Basal level of (p)ppGpp measurement in KP32 (spoT1 rumA::CAT) mutant grown in MOPS glucose at (A) $37^{\circ} \mathrm{C} ;(\mathbf{B}) 30^{\circ} \mathrm{C}$ and $(\mathbf{C})$ in response to isoleucine starvation.

Fig. 5: Plasmid mediated low to moderate level expression of RelA in the spoT1 mutant background (KP31) recapitulates the growth phenotypes of $\operatorname{rumA::CAT}$ spoT1 mutant, KP32: 5A: (Ia) Growth of pGB2 relA transformants of WT and spoT1 mutant on minimal agar plate at $30^{\circ} \mathrm{C}$, (Ib) Growth curve of the pGB2 and pGB2 RelA transformants of spoT1 and spoT1 rumA::CAT mutants was measured in minimal broth at $37^{\circ} \mathrm{C}$. The growth curves were plotted from three independent experiments, (Ic) Growth of pGB2 relA transformants of WT and spoT1 mutant on minimal agar plate supplemented with 3 -ATat $37^{\circ} \mathrm{C}$. (IIa, IIb) TLC measurement and quantitation respectively of basal level of (p)ppGpp in KP32 (spoT1 rumA::CAT), MG1655/pGB2 relA and KP31 (spoT1)/ pGB2 relA in cells grown in MOPS glucose medium, (III) Quantification of RelA protein in KP32, MG1655/ pGB2 relA and spoT1 mutant (KP31)/ pGB2 relA by Western blot; (5B): Moderate level expression of RelA-CTD (pTE22 (RelA-CTD1)) and (pTE23 (RelA-CTD2)) selectively inhibits growth of the spoT1 mutant: (Ia) Growth phenotype of relA ${ }^{+}$strains, MG1655 and KP31 (spoT1), transformed with following plasmids - control (pBAD18Kan), pTE6 (pBAD18Kan RelA), 
pTE22 (RelA-CTD1) and pTE23 (RelA-CTD2) on minimal medium and minimal medium supplemented with 3-AT; (Ib) growth curve measurement of the same set of strains as in Ia in minimal broth at $37^{\circ} \mathrm{C}$. The growth curves were plotted from three independent experiments; (5C) Severe growth retardation phenotype of transformants of KP31 (spoT1)/pALS10 on LA + Amp plate. The plate was incubated at $30^{\circ} \mathrm{C}$.

\section{Fig. 6: Obliteration of the relAl mutation-mediated growth inhibitory effects of overexpression of RelA-CTD in spoT1 mutant is reversed by spoT202 mutation}

(Ia) Growth on minimal medium and minimal medium supplemented with 3-AT of relA1 mutant strains KP52 (MG1655 relA1) and KP53 (MG1655 relAlspoT1) transformed with plasmids - control (pBAD18Kan), pTE6 (pBAD18Kan RelA), pTE22 (RelA-CTD1) and pTE23 (RelA-CTD2); (Ib) Growth curve of transformants of same set of strains as in Ia was measured in Minimal broth at $37^{\circ} \mathrm{C}$. The growth curves were plotted from three independent experiments. (IIa) Growth phenotype of pTE22 (RelA-CTD1) and pTE23 (RelA-CTD2) transformants of KP56 (relA1 spoT202) is significantly retarded in comparison to that of control plasmid, pBAD18Kan and the CTD plasmid transformants of KP57. (IIb) Growth curve measurements of the pTE22, pTE23 plasmid transformants of KP56 and KP57. The growth curves were plotted from three independent experiments. $(\mathbf{C})$ : Severe growth effects of overexpression of RelA-CTD1 (pTE22) (I), and pTE23 (RelA-CTD2) (II) in relA spoT202 mutant KT58. The growth of the pBAD18Kan transformants of each of the mutant was obvious on the plates within $16 \mathrm{hrs}$. of incubation in contrast to that of pTE22/pTE23 transformants. The plates were photographed after $24 \mathrm{hrs}$ of incubation at $37^{\circ} \mathrm{C}$.

\section{Legends to Supplementary figures}


Fig. S1: Measurement of growth of relA $^{+}$and spoT1 mutants of MC4100 and MG1655 strains respectively in minimal broth at $37^{\circ} \mathbf{C}$. Growth curve is representative of three independent experiments.

Fig. S2: pTE18 (pBR322 spoT ${ }^{+}$) plasmid suppresses slow growth and stringent response defect of KP8 [MC4100 (relA ${ }^{+}$spoT1 rumA::CAT)]. (A) Growth on minimal agar and (B) on amino acid starvation plate (Minimal agar $+3-\mathrm{AT})$. The plates were incubated at $37^{\circ} \mathrm{C}$.

Fig. S3: Differential effects of spoT1 mutation on stringent response in strains MG1655 and MC4100 (A) spoT1 allele suppresses the stringent response defect of the relA1 mutation in KP53 (MG1655 spoT1relA1) and promotes its growth on minimal medium supplemented with 3-AT, whereas the growth of the strain in MC4100 containing the same combination of alleles is sensitive to 3-AT; (B) complementation of the defective stringent response of MC4100 (relA1 spoT1) with the plasmid pTE6 (pBAD18Kan RelA) but not by pTE22 (RelA-CTD1) on minimal plate supplemented with 3-AT.

Fig. S4: Growth Phenotypes of spoT202 mutant derivatives of $\mathrm{relA}^{+}$and $\mathrm{relAl}$ strains:

A: Slow Growth of spoT202 relA ${ }^{+}$strain (KP58) on LA agar plate in comparison to those of relAl mutant (KP56). The plates were incubated at $37^{\circ} \mathrm{C}$ and photographed after $16 \mathrm{hrs}$. of incubation. B: The stringent response of relA1 spoT202 mutant was assessed on minimal agar plate supplemented with $15 \mathrm{mM}$ 3-AT. 1 through 5 are spoT202 relA1 mutants (KP56 being the representative) that grow on the histidine starvation plate due to high intracellular ppGpp levels and 6 through 8 are three independent isogenic relAl spo $T^{+}$derivatives (KP57 being the representative) that do not grow. 
Table1: Strains and plasmids used in this study

\begin{tabular}{|c|c|c|}
\hline $\begin{array}{c}E . \text { coli } \\
\text { strains/Plasmids }\end{array}$ & Relevant genotype/Description & Reference/Source \\
\hline \multicolumn{3}{|c|}{ Strains } \\
\hline MG1655 & $\lambda^{-}, r p h-1$ & lab collection \\
\hline MC4100 KP & $\begin{array}{l}F^{-} \text {araD139 (argF-lac)U169 rpsL150 deoC1 relA1 thiA ptsF25 } \\
\text { flbB5301 rbsR }\end{array}$ & lab collection \\
\hline DY330 & W3110 $\Delta l a c U 169$ gal490 $\lambda$ cI857 $\Delta($ cro-bioA $)$ & (Yu et al., 2000) \\
\hline CAG12182 & $\lambda, c y s 13152:: \operatorname{Tn} 10 \mathrm{kan}, \mathrm{rph} 1$ & (Singer et al., 1989) \\
\hline JW2756-1 & $\begin{array}{l}\Delta(\text { araD-araB }) 567, \text { AlacZ4787(::rrnB-3), } \lambda^{-}, \Delta \text { rumA783::kan, rph-1, } \\
\Delta(\text { rhaD-rhaB }) 568, h s d R 514\end{array}$ & $\begin{array}{l}\text { Keio Collection, } \\
\text { (Baba et al., 2006)) }\end{array}$ \\
\hline JW2757-1 & $\begin{array}{l}\Delta(\text { araD-araB }) 567, \text { AlacZ4787(::rrnB-3), } \lambda^{-}, \text {, barA784::kan, rph-1, } \\
\Delta(\text { rhaD-rhaB }) 568, h s d R 514\end{array}$ & $\begin{array}{l}\text { Keio Collection, } \\
\text { (Baba et al., 2006) }\end{array}$ \\
\hline JW2758-5 & $\begin{array}{l}\Delta(\text { araD-araB }) 567, \Delta \text { lacZ4787(::rrnB-3), } \lambda^{-}, \Delta g u d D 785:: k a n, r p h-1, \\
\Delta(\text { rhaD-rhaB }) 568, \text { hsdR514 }\end{array}$ & $\begin{array}{l}\text { Keio Collection, } \\
\text { (Baba } \text { et al., 2006) }\end{array}$ \\
\hline CF17960 & MG1655 ArelA256 spoT202 (spoT T78I) zib563::Tn10 & M. Cashel lab \\
\hline CF17961 & MG1655 ArelA256 spoT203 (spoT R140C) zib563::Tn10 & M. Cashel lab \\
\hline KP2 & MC4100 relA ${ }^{+}$cysI3152::Tn10kan (transduction from CAG12182 ) & This study \\
\hline KP4 & MC4100 $\mathrm{relA}^{+}$cysI $I^{+}$ & This study \\
\hline KP8 & MC4100 relA ${ }^{+}$rumASalI::CAT & This study \\
\hline KP9 & MC4100 relA ${ }^{+}$rumAMluI::CAT & This study \\
\hline KP11 & MC4100KP spoT1 $\triangle p y r E 748:: K A N$ (transduction fromJW3617-1) & This study \\
\hline KP19 & MC4100 relA::IS2rumASalI::CAT & This study \\
\hline KP24 & MG1655 relA ${ }^{+}$rumASalI::CAT & This study \\
\hline KP25 & MG1655relA ${ }^{+}$rumAMluI::CAT & This study \\
\hline KP29 & MG1655 spoT1 $\triangle p y r E 748:: K A N$ (transduction from KP11) & This study \\
\hline KP30 & KP24 spoT1 ApyrE748::KAN (transduction from KP11) & This study \\
\hline KP31 & MG1655 spoT1pyrE ${ }^{+}$ & This study \\
\hline KP32 & KP30 spoT1 pyrE & This study \\
\hline KP51 & MC4100KP relA1 barA::CAT & This study \\
\hline KP52 & MG1655 relA1 barA::CAT (transduction from KP51) & This study \\
\hline KP53 & $\begin{array}{l}\text { MG1655relA1 spoT1barA::CAT [transduction from KP51 } \\
\text { (MC4100KP barA::CAT)] }\end{array}$ & This study \\
\hline KP54 & MG1655spoT1relA $\triangle P v u \mathrm{II}:: C A T$ & This study \\
\hline KP55 & MG1655 relA101 (relA $\triangle P v u \mathrm{II}:: C A T)$ & This study \\
\hline KP56 & KP52 spoT202 zib563::Tn10 (transduction from CF17960) & This study \\
\hline KP57 & KP52 zib563::Tn10 (transduction from CF17960) & This study \\
\hline KP58 & MG1655 spoT202 zib563::Tn10 (transduction from CF17960) & This study \\
\hline KP59 & MC4100 relA ${ }^{+}$rumASalI:: TAC & This study \\
\hline KP60 & MG1655 relA ${ }^{+}$rumASalI:: TAC & This study \\
\hline \multicolumn{3}{|c|}{$\begin{array}{r}\text { Plasmids } \\
\end{array}$} \\
\hline
\end{tabular}




\begin{tabular}{|c|c|c|}
\hline pBAD18KAN & Cloning Vector Kan ${ }^{\mathrm{r}}$ ColE1 replicon & (Guzman et al., 1995) \\
\hline pBlueScriptKS & Cloning Vector $\mathrm{Amp}^{\mathrm{r}}$ ColE1 replicon & Stratagene,USA \\
\hline pBBR1MCS2 & Cloning vector $\operatorname{Kan}^{\mathrm{r}}$ p $15 \mathrm{~A}$ replicon & (Kovach et al., 1995) \\
\hline pACYC184 & Cloning vector $\mathrm{Cm}^{\mathrm{r},} \mathrm{Tet}^{\mathrm{r}}$ & (Bartolome et al., 1991) \\
\hline pBR322 & Cloning vectorAmp ${ }^{\mathrm{r},} \mathrm{Tet}^{\mathrm{r}}$ & (Bolivar, 1978) \\
\hline pKD46 & Recombineering vector, Ori ${ }^{\text {ts }} A m p^{\text {r }}$ repA101 replicon & $\begin{array}{l}\text { (Datsenko \& Wanner, } \\
\text { 2000) }\end{array}$ \\
\hline pBG2 & pSC101 derived cloning vector, $\mathrm{Spc}^{\mathrm{r}}$ & (Churchward et al., 1984) \\
\hline pGB2 relA & pGB2 containing inducible full-length (743-aa) RelA & M. Cashel \\
\hline pALS10 & $\begin{array}{l}\mathrm{P}_{\text {tac }}: \text { relA } \text { lacl }^{\mathrm{q}} \text {, ColE1 replicon, } \mathrm{Ap}^{\mathrm{r}} \text {, inducible full-length (743-aa) } \\
\text { RelA }\end{array}$ & (Svitil et al., 1993), \\
\hline pALS13 & As pALS10 but relA' 455-aa RelA fragment & (Svitil et al., 1993) \\
\hline pALS14 & $\begin{array}{l}\text { As pALS10 but relA' 331-aa RelA fragment; catalytically inactive } \\
\text { control RelA }\end{array}$ & (Svitil et al., 1993) \\
\hline pTE6 & $\begin{array}{l}3167 \text { bp long continuous segment bearing relA gene }(2289 \mathrm{bp}) \text { with } \\
\text { its native promotersin the upstream rumA DNA }(11 \text { bp)cloned at } \\
\text { EcoRI - KpnI fragment in pBAD18Kan. }\end{array}$ & This study \\
\hline pTE14 & $\begin{array}{l}1529 \text { bp rumA amplicon }+178 \text { bp upstream barA DNA cloned at } \\
\text { EcoRI - KpnI of pBBR1MCS } 2\end{array}$ & This study \\
\hline pTE15 & pTE14 containing CATcassette cloned at unique SalI site in rumA & This study \\
\hline pTE16 & $\begin{array}{l}\text { pTE1 } \triangle \mathrm{Bgl} \text { containing } C A T \text { cassette cloned at unique } M l u \mathrm{I} \text { site in } \\
\text { rumA }\end{array}$ & This study \\
\hline pTE17 & $\begin{array}{l}\text { pTE1 } \triangle \mathrm{Bgl} \text { containing } C A T \text { cassette cloned at unique } B g l \mathrm{II} \text { site in } \\
\text { barA }\end{array}$ & This study \\
\hline pTE18 & pBR322 subcloned spoT $T^{+}$ & This study \\
\hline pTE19 & $\begin{array}{l}1529 \text { bp rumA amplicon }+178 \text { bp upstream barA DNA cloned at } \\
\text { EcoRI - KpnI of pBlueScriptKS }\end{array}$ & This study \\
\hline pTE20 & pTE19 containing $K A N$ cassette cloned at unique SalI site in rumA & This study \\
\hline pTE22 & $\begin{array}{l}P v u \text { II restriction enzyme digestion of pTE6 plasmid followed by } \\
\text { intramolecular ligation generates in-frame N-terminal 1-119 amino } \\
\text { acids fusion with entire C-terminal domain of RelA from 455- } \\
744 \text { amino acids (pRelA-CTD1) }\end{array}$ & This study \\
\hline pTE23 & $\begin{array}{l}\text { Recombineering-mediated repair of PvuII-linearized pTE22 DNA } \\
\text { by PCR product containing in-frame N-terminal 1-119 amino acids } \\
\text { fusion with C-terminal domain of RelA from 406-744 amino acids } \\
\text { (pRelA-CTD2) }\end{array}$ & This study \\
\hline
\end{tabular}

Table 2: List of Primers used in this work

\begin{tabular}{|c|c|}
\hline Primer Name & Primer sequence $5^{\prime} \rightarrow 3^{\prime}$ \\
\hline RumAF1 & TTAGAATTCGGATCCAGTTGACGCTGCA \\
\hline RumAF3 & AATGGATCCTGCTGCGTAGTGGGAA \\
\hline RumARKpn 1 & AATGGTACCGACCAGACCTGCCGAA \\
\hline BarAF & CGTGGTACCTTGGCGAGACTTTCTCA \\
\hline RelAF & CTGGAATTCGCAGGTCTGGTCCCTA \\
\hline RelAR & CGT $\overline{\overline{G G T A C C} G A G C A A A T T T C G G C C T A}$ \\
\hline CATFSalI $^{\mathrm{a}}$ & TTAGTCGACATGAGACGTTGATCGGCACG \\
\hline CATRSalI $^{\mathrm{a}}$ & AATGTCGACATTCAGGCGTAGCACCAGGC \\
\hline CATFMB $^{\mathrm{a}}$ & TTAACGCGTAGATCTATGAGACGTTGATCGGCACG \\
\hline CATRMB $^{\mathrm{a}}$ & AATACGCGTAGATCTATTCAGGCGTAGCACCAGGC \\
\hline
\end{tabular}




\begin{tabular}{|l|l|}
\hline KANLeftSalI & TTAGTCGACACGTTGTGTCTCAAAATCTC \\
\hline KAN RightSalI & AATGTCGACTACAACCAATTAACCAATTCTG \\
\hline RelART Left & ACTACCACGCGACCGTTATC \\
\hline RelART Right & GGGCGACCAGATTGAAATTA \\
\hline GAPDH RT Left & AGGTCTGATGACCACCGTTC \\
\hline GAPDHRT Right & AGTAACCGGTTTCGTTGTCG \\
\hline RelA Fusion primer Lft & GTAAAGACGTACACCAGCTGGCGGATCG \\
\hline RelA fusion primer Rgt & CGATCCGCCAGCTGGTGTACGTCTTAC \\
\hline Poteete et al., 2006 ) & \\
\hline
\end{tabular}

\section{References:}

An, G., J. Justesen, R.J. Watson \& J.D. Friesen, (1979) Cloning the spoT gene of Escherichia coli: identification of the spoT gene product. J Bacteriol 137: 1100-1110.

Arenz, S., M. Abdelshahid, D. Sohmen, R. Payoe, A.L. Starosta, O. Berninghausen, V. Hauryliuk, R. Beckmann \& D.N. Wilson, (2016) The stringent factor RelA adopts an open conformation on the ribosome to stimulate ppGpp synthesis. Nucleic acids research 44: 6471-6481.

Atkinson, G.C., T. Tenson \& V. Hauryliuk, (2011) The RelA/SpoT homolog (RSH) superfamily: distribution and functional evolution of ppGpp synthetases and hydrolases across the tree of life. PloS one 6: e23479.

Avarbock, A., D. Avarbock, J.-S. Teh, M. Buckstein, Z.-m. Wang \& H. Rubin, (2005) Functional regulation of the opposing (p) ppGpp synthetase/hydrolase activities of RelMtb from Mycobacterium tuberculosis. Biochemistry 44: 9913-9923.

Baba, T., T. Ara, M. Hasegawa, Y. Takai, Y. Okumura, M. Baba, K.A. Datsenko, M. Tomita, B.L. Wanner \& H. Mori, (2006) Construction of Escherichia coli K-12 in-frame, single-gene knockout mutants: the Keio collection. Molecular systems biology 2.

Bartolome, B., Y. Jubete, E. Martínez \& F. de la Cruz, (1991) Construction and properties of a family of pACYC184-derived cloning vectors compatible with pBR322 and its derivatives. Gene 102: 75-78.

Bolivar, F., (1978) Construction and characterization of new cloning vehicles III. Derivatives of plasmid pBR322 carrying unique Eco RI sites for selection of Eco RI generated recombinant DNA molecules. Gene 4: 121-136.

Brown, A., I.S. Fernández, Y. Gordiyenko \& V. Ramakrishnan, (2016) Ribosome-dependent activation of stringent control. Nature 534: 277-280.

Brown, D.R., G. Barton, Z. Pan, M. Buck \& S. Wigneshweraraj, (2014) Nitrogen stress response and stringent response are coupled in Escherichia coli. Nature comm unications 5:4115.

Brown, L., D. Gentry, T. Elliott \& M. Cashel, (2002) DksA affects ppGpp induction of RpoS at a translational level. Journal of bacteriology 184: 4455-4465.

Butland, G., M. Babu, J.J. Diaz-Mejia, F. Bohdana, S. Phanse, B. Gold, W. Yang, J. Li, A.G. Gagarinova, O. Pogoutse, H. Mori, B.L. Wanner, H. Lo, J. Wasniewski, C. Christopolous, M. Ali, P. Venn, A. Safavi-Naini, N. Sourour, S. Caron, J.Y. Choi, L. Laigle, A. Nazarians-Armavil, A. Deshpande, S. Joe, K.A. Datsenko, N. Yamamoto, B.J. Andrews, C. Boone, H. Ding, B. Sheikh, G. MorenoHagelseib, J.F. Greenblatt \& A. Emili, (2008) eSGA: E. coli synthetic genetic array analysis. Nat Methods 5: 789-795.

Cashel, M., (1994) Detection of (p) ppGpp accumulation patterns in Escherichia coli mutants. Methods Mol. Genet 3: 341-356.

Cashel, M., D. Gentry, V. Hernandez \& D. Vinella, (1996) The Stringent Response. In: Escherichia coli and Salmonella Cellular and Molecular Biology.

Churchward, G., D. Belin \& Y. Nagamine, (1984) A pSC101-derived plasmid which shows no sequence homology to other commonly used cloning vectors. Gene 31: 165-171.

Datsenko, K.A. \& B.L. Wanner, (2000) One-step inactivation of chromosomal genes in Escherichia coli K-12 using PCR products. Proceedings of the National Academy of Sciences 97: 6640-6645. 
Dozot, M., R.A. Boigegrain, R.M. Delrue, R. Hallez, S. Ouahrani-Bettache, I. Danese, J.J. Letesson, X. De Bolle \& S. Köhler, (2006) The stringent response mediator Rsh is required for Brucella melitensis and Brucella suis virulence, and for expression of the type IV secretion system virB. Cellular microbiology 8: 1791-1802.

Ferenci, T., H.F. Galbiati, T. Betteridge, K. Phan \& B. Spira, (2011) The constancy of global regulation across a species: the concentrations of ppGpp and RpoS are strain-specific in Escherichia coli. $B M C$ microbiology 11: 62.

Fernández-Coll, L. \& M. Cashel, (2019) Using Microtiter Dish Radiolabeling for Multiple In Vivo Measurements Of Escherichia coli (p) ppGpp Followed by Thin Layer Chromatography. JoVE (Journal of Visualized Experiments): e59595.

Fiil, N.P., B.M. Willumsen, J. Friesen \& K. von Meyenburg, (1977) Interaction of alleles of therelA, relC andspoT genes inEscherichia coli: Analysis of the interconversion of GTP, ppGpp and pppGpp. Molecular and General Genetics MGG 150: 87-101.

Gaca, A.O., C. Colomer-Winter \& J.A. Lemos, (2015) Many means to a common end: the intricacies of (p) ppGpp metabolism and its control of bacterial homeostasis. Journal of bacteriology: JB. 02577-02514.

Geiger, T., C. Goerke, M. Fritz, T. Schäfer, K. Ohlsen, M. Liebeke, M. Lalk \& C. Wolz, (2010) Role of the (p) ppGpp synthase RSH, a RelA/SpoT homolog, in stringent response and virulence of Staphylococcus aureus. Infection and immunity 78: 1873-1883.

Gentry, D.R. \& M. Cashel, (1996) Mutational analysis of the Escherichia coli spoT gene identifies distinct but overlapping regions involved in ppGpp synthesis and degradation. Molecular microbiology 19: 1373-1384.

Gratani, F.L., P. Horvatek, T. Geiger, M. Borisova, C. Mayer, I. Grin, S. Wagner, W. Steinchen, G. Bange \& A. Velic, (2018) Regulation of the opposing (p) ppGpp synthetase and hydrolase activities in a bifunctional RelA/SpoT homologue from Staphylococcus aureus. PLoS genetics 14: e1007514.

Gropp, M., Y. Strausz, M. Gross \& G. Glaser, (2001) Regulation of Escherichia coli RelA requires oligomerization of the C-terminal domain. Journal of bacteriology 183: 570-579.

Guzman, L.M., D. Belin, M.J. Carson \& J. Beckwith, (1995) Tight regulation, modulation, and highlevel expression by vectors containing the arabin ose PBAD promoter. J Bacteriol 177: 41214130.

Haseltine, W.A. \& R. Block, (1973) Synthesis of guanosine tetra-and pentaphosphate requires the presence of a codon-specific, uncharged transfer ribonucleic acid in the acceptor site of ribosomes. Proceedings of the National Academy of Sciences 70: 1564-1568.

Hauryliuk, V., G.C. Atkinson, K.S. Murakami, T. Tenson \& K. Gerdes, (2015) Recent functional insights into the role of (p) ppGpp in bacterial physiology. Nature Reviews Microbiology 13: 298.

Hernandez, V.J. \& H. Bremer, (1991) Escherichia coli ppGpp synthetase II activity requires spoT. Journal of Biological Chemistry 266: 5991-5999.

lyer, S., D. Le, B.R. Park \& M. Kim, (2018) Distinct mechanisms coordinate transcription and translation under carbon and nitrogen starvation in Escherichia coli. Nature microbiology 3 : 741-748.

Jain, V., M. Kumar \& D. Chatterji, (2006a) ppGpp: stringent response and survival. JOURNAL OF MICROBIOLOGY-SEOUL- 44: 1.

Jain, V., R. Saleem-Batcha, A. China \& D. Chatterji, (2006b) Molecular dissection of the mycobacterial stringent response protein Rel. Protein Science 15: 1449-1464.

King, T., A. Ishihama, A. Kori \& T. Ferenci, (2004) A regulatory trade-off as a source of strain variation in the species Escherichia coli. J Bacteriol 186: 5614-5620.

Kovach, M.E., P.H. Elzer, D.S. Hill, G.T. Robertson, M.A. Farris, R.M. Roop, 2nd \& K.M. Peterson, (1995) Four new derivatives of the broad-host-range cloning vector pBBR1MCS, carrying different antibiotic-resistance cassettes. Gene 166: 175-176. 
Kudrin, P., I. Dzhygyr, K. Ishiguro, J. Beljantseva, E. Maksimova, S.R.A. Oliveira, V. Varik, R. Payoe, A.L. Konevega \& T. Tenson, (2018) The ribosomal A-site finger is crucial for binding and activation of the stringent factor RelA. Nucleic acids research 46: 1973-1983.

Kushwaha, G.S., G. Bange \& N.S. Bhavesh, (2019) Interaction studies on bacterial stringent response protein RelA with uncharged tRNA provide evidence for its prerequisite complex for ribosome binding. Current genetics 65: 1173-1184.

Laffler, T. \& J. Gallant, (1974a) spoT, a new genetic locus involved in the stringent response in E. coli. Cell 1: 27-30.

Laffler, T. \& J.A. Gallant, (1974b) Stringent control of protein synthesis in E. coli. Cell 3: 47-49.

Loveland, A.B., E. Bah, R. Madireddy, Y. Zhang, A.F. Brilot, N. Grigorieff \& A. A. Korostelev, (2016) Ribosome RelA structures reveal the mechanism of stringent response activation. Elife $\mathbf{5}$ : e17029.

Mechold, U., M. Cashel, K. Steiner, D. Gentry \& H. Malke, (1996) Functional analysis of a relA/spoT gene homolog from Streptococcus equisimilis. Journal of bacteriology 178: 1401-1411.

Metzger, S., I.B. Dror, E. Aizenman, G. Schreiber, M. Toone, J. Friesen, M. Cashel \& G. Glaser, (1988) The nucleotide sequence and characterization of the relA gene of Escherichia coli. Journal of Biological Chemistry 263: 15699-15704.

Metzger, S., G. Schreiber, E. Aizenman, M. Cashel \& G. Glaser, (1989) Characterization of the relA1 mutation and a comparison of relA1 with new relA null alleles in Escherichia coli. J Biol Chem 264: 21146-21152.

Miller, J.H., (1992) A short course in bacterial genetics, A Laboratory Manual and Handbook for Escherichia coli and Related Bacteria. Cold Spring Harbor Laboratory Press.

Montero, M., M. Rahimpour, A.M. Viale, G. Almagro, G. Eydallin, Á. Sevilla, M. Cánovas, C. Bernal, A.B. Lozano \& F.J. Muñoz, (2014) Systematic Production of Inactivating and Non-Inactivating Suppressor Mutations at the relA Locus That Compensate the Detrimental Effects of Complete spoT Loss and Affect Glycogen Content in Escherichia coli. PloS one 9: e106938.

Nakagawa, A., T. Oshima \& H. Mori, (2006) Identification and characterization of a second, inducible promoter of relA in Escherichia coli. Genes \& genetic systems 81: 299-310.

Ochi, K., J. Kandala \& E. Freese, (1981) Initiation of Bacillus subtilis sporulation by the stringent response to partial amino acid deprivation. Journal of Biological Chemistry 256: 6866-6875.

Persaud, C., Y. Lu, A. Vila-Sanjurjo, J.L. Campbell, J. Finley \& M. O'Connor, (2010) Mutagenesis of the modified bases, $m^{5} \mathrm{U} 1939$ and $\psi 2504$, in Escherichia coli 23 S rRNA. Biochemical and biophysical research communications 392: 223-227.

Poole, K., (2012) Bacterial stress responses as determinants of antimicrobial resistance. J Antimicrob Chemother 67: 2069-2089.

Poteete, A.R., C. Rosadini \& C. StPierre, (2006) Gentamicin and other cassettes for chromosomal gene replacement in Escherichia coli. Biotechniques 41: 261.

Potrykus, K. \& M. Cashel, (2008) (p)ppGpp: still magical? Annu.Rev. Microbiol. 62: 35-51.

Ronneau, S. \& R. Hallez, (2019) Make and break the alarmone: regulation of (p) ppGpp synthetase/hydrolase enzymes in bacteria. FEMS microbiology reviews.

Sambrook, J., Fritsch, E.F., and Maniatis, T. , (1989) Molecular Cloning, A Laboratory Manual.

Sanchez-Vazquez, P., C.N. Dewey, N. Kitten, W. Ross \& R.L. Gourse, (2019) Genome-wide effects on Escherichia coli transcription from ppGpp binding to its two sites on RNA polymerase. Proceedings of the National Academy of Sciences 116: 8310-8319.

Sarubbi, E., K.E. Rudd \& M. Cashel, (1988) Basal ppGpp level adjustment shown by new spoT mutants affect steady state growth rates and rrnA ribosomal promoter regulation in Escherichia coli. Mol Gen Genet 213: 214-222.

Schofield, W.B., M. Zimmermann-Kogadeeva, M. Zimmermann, N.A. Barry \& A.L. Goodman, (2018) The stringent response determines the ability of a commensal bacterium to survive starvation and to persist in the gut. Cell host \& microbe 24: 120-132. e126. 
Schreiber, G., S. Metzger, E. Aizenman, S. Roza, M. Cashel \& G. Glaser, (1991) Overexpression of the relA gene in Escherichia coli. Journal of Biological chemistry 266: 3760-3767.

Seyfzadeh, M., J. Keener \& M. Nomura, (1993) spoT-dependent accumulation of guanosine tetraphosphate in response to fatty acid starvation in Escherichia coli. Proceedings of the National Academy of Sciences 90: 11004-11008.

Shyp, V., S. Tankov, A. Ermakov, P. Kudrin, B.P. English, M. Ehrenberg, T. Tenson, J. Elf \& V. Hauryliuk, (2012) Positive allosteric feedback regulation of the stringent response enzyme RelA by its product. EMBO reports 13: 835-839.

Silva, A.J. \& J.A. Benitez, (2006) A Vibrio cholerae relaxed (relA) mutant expresses major virulence factors, exhibits biofilm formation and motility, and colonizes the suckling mouse intestine. $J$ Bacteriol 188: 794-800.

Singer, M., T. Baker, G. Schnitzler, S. Deischel, M. Goel, W. Dove, K. Jaacks, A. Grossman, J. Erickson \& C.A. Gross, (1989) A collection of strains containing genetically linked alternating antibiotic resistance elements for genetic mapping of Escherichia coli. Microbiological reviews 53: 124.

Sinha, A.K., K.S. Winther, M. Roghanian \& K. Gerdes, (2019) Fatty acid starvation activates RelA by depleting lysine precursor pyruvate. bioRxiv: 635748 .

Spira, B. \& T. Ferenci, (2008) Alkaline phosphatase as a reporter of $\sigma S$ levels and rpos polymorphisms in different E. coli strains. Archives of microbiology 189: 43-47.

Spira, B., X. Hu \& T. Ferenci, (2008) Strain variation in ppGpp concentration and RpoS levels in laboratory strains of Escherichia coli K-12. Microbiology 154: 2887-2895.

Spira, B. \& K. Ospino, (2020) Diversity in E. coli (p) ppGpp Levels and Its Consequences. Frontiers in Microbiology 11: 1759.

Spira, B., N. Silberstein \& E. Yagil, (1995) Guanosine 3', 5'-bispyrophosphate (ppGpp) synthesis in cells of Escherichia coli starved for Pi. Journal of bacteriology 177: 4053-4058.

Steinchen, W. \& G. Bange, (2016) The magic dance of the alarmones (p) ppGpp. Molecular microbiology 101: 531-544.

Svitil, A., M. Cashel \& J. Zyskind, (1993) Guanosine tetraphosphate inhibits protein synthesis in vivo. A possible protective mechanism for starvation stress in Escherichia coli. Journal of Biological Chemistry 268: 2307-2311.

Syal, K., H. Joshi, D. Chatterji \& V. Jain, (2015) Novel pppGpp binding site at the C-terminal region of the Rel enzyme from Mycobacterium smegmatis. The FEBS journal 282: 3773-3785.

Takada, H., M. Roghanian, J. Caballero-Montes, K. Van Nerom, S. Jimmy, P. Kudrin, F. Trebini, R. Murayama, G. Akanuma \& A. Garcia-Pino, (2021) Ribosome association primes the stringent factor Rel for tRNA-dependent locking in the A-site and activation of (p) ppGpp synthesis. Nucleic Acids Research 49: 444-457.

Tamman, H., K. Van Nerom, H. Takada, N. Vandenberk, D. Scholl, Y. Polikanov, J. Hofkens, A. Talavera, V. Hauryliuk \& J. Hendrix, (2019) Nucleotide-mediated allosteric regulation of bifunctional Rel enzymes. bioRxiv: 670703.

Tedin, K. \& F. Norel, (2001) Comparison of $\Delta$ relA strains of Escherichia coli and Salmonella enterica serovar Typhimurium suggests a role for $\mathrm{ppGpp}$ in attenuation regulation of branched-chain amino acid biosynthesis. Journal of bacteriology 183: 6184-6196.

Tosa, T. \& L.I. Pizer, (1971) Effect of serine hydroxamate on the growth of Escherichia coli. Journal of bacteriology 106: 966-971.

Turnbull, K.J., L. Dzhygyr, S. Lindemose, V. Hauryliuk \& M. Roghanian, (2019) Intramolecular interactions dominate the autoregulation of Escherichia coli stringent factor RelA. Frontiers in Microbiology 10: 1966.

Vinella, D., C. Albrecht, M. Cashel \& R. D'Ari, (2005) Iron limitation induces SpoT-dependent accumulation of ppGpp in Escherichia coli. Molecular microbiology 56: 958-970. 
Wang, J., Y. Tian, Z. Zhou, L. Zhang, W. Zhang, M. Lin \& M. Chen, (2016) Identification and functional analysis of RelA/SpoT Homolog (RSH) genes in Deinococcus radiodurans. Journal of microbiology and biotechnology 26: 2106-2115.

Wendrich, T.M., G. Blaha, D.N. Wilson, M.A. Marahiel \& K.H. Nierhaus, (2002) Dissection of the mechanism for the stringent factor RelA. Mol Cell 10: 779-788.

Wilkinson, R.C., L.E. Batten, N.J. Wells, P.C. Oyston \& P.L. Roach, (2015) Biochemical studies on Francisella tularensis RelA in (p) ppGpp biosynthesis. Bioscience reports 35.

Winther, K.S., M. Roghanian \& K. Gerdes, (2018) Activation of the stringent response by loading of RelA-tRNA complexes at the ribosomal A-site. Molecular cell 70: 95-105. e104.

Xiao, H., M. Kalman, K. Ikehara, S. Zemel, G. Glaser \& M. Cashel, (1991) Residual guanosine 3', 5'bispyrophosphate synthetic activity of relA null mutants can be eliminated by spoT null mutations. Journal of Biological Chemistry 266: 5980-5990.

Yang, X. \& E.E. Ishiguro, (2001) Involvement of the $\mathrm{N}$ terminus of ribosomal protein $\mathrm{L} 11$ in regulation of the RelA protein of Escherichia coli. Journal of bacteriology 183: 6532-6537.

Yu, D., H.M. Ellis, E.C. Lee, N.A. Jenkins, N.G. Copeland \& D.L. Court, (2000) An efficient recombination system for chromosome engineering in Escherichia coli. Proc Natl Acad SciU S A 97: 5978-5983.

Yusupov, M.M., G.Z. Yusupova, A. Baucom, K. Lieberman, T.N. Earnest, J. Cate \& H.F. Noller, (2001) Crystal structure of the ribosome at $5.5 \AA$ resolution. science 292: 883-896.

Zhu, M. \& X. Dai, (2019) Growth suppression by altered (p) ppGpp levels results from non-optimal resource allocation in Escherichia coli. Nucleic acids research 47: 4684-4693. 
Fig.1

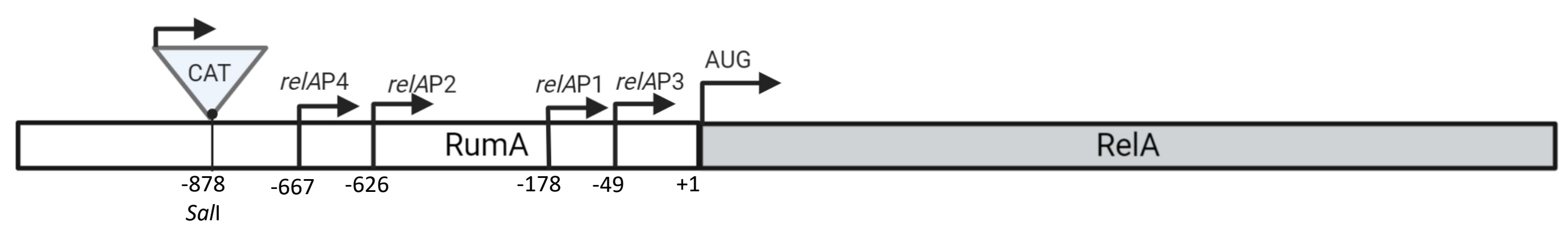


Fig. 2

A.

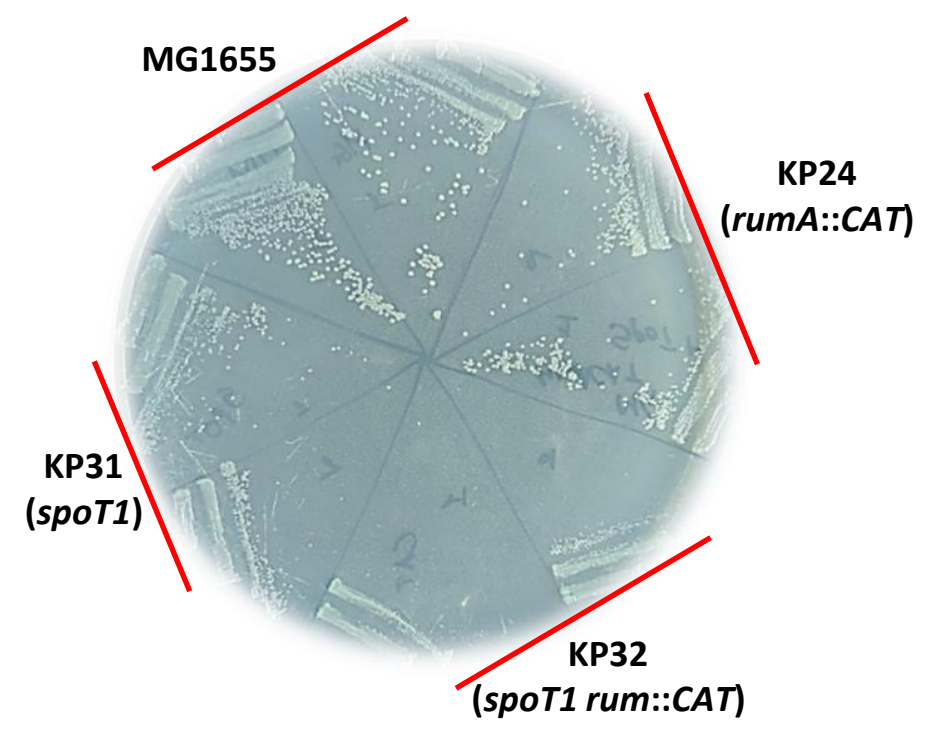

C. ${ }^{1}$

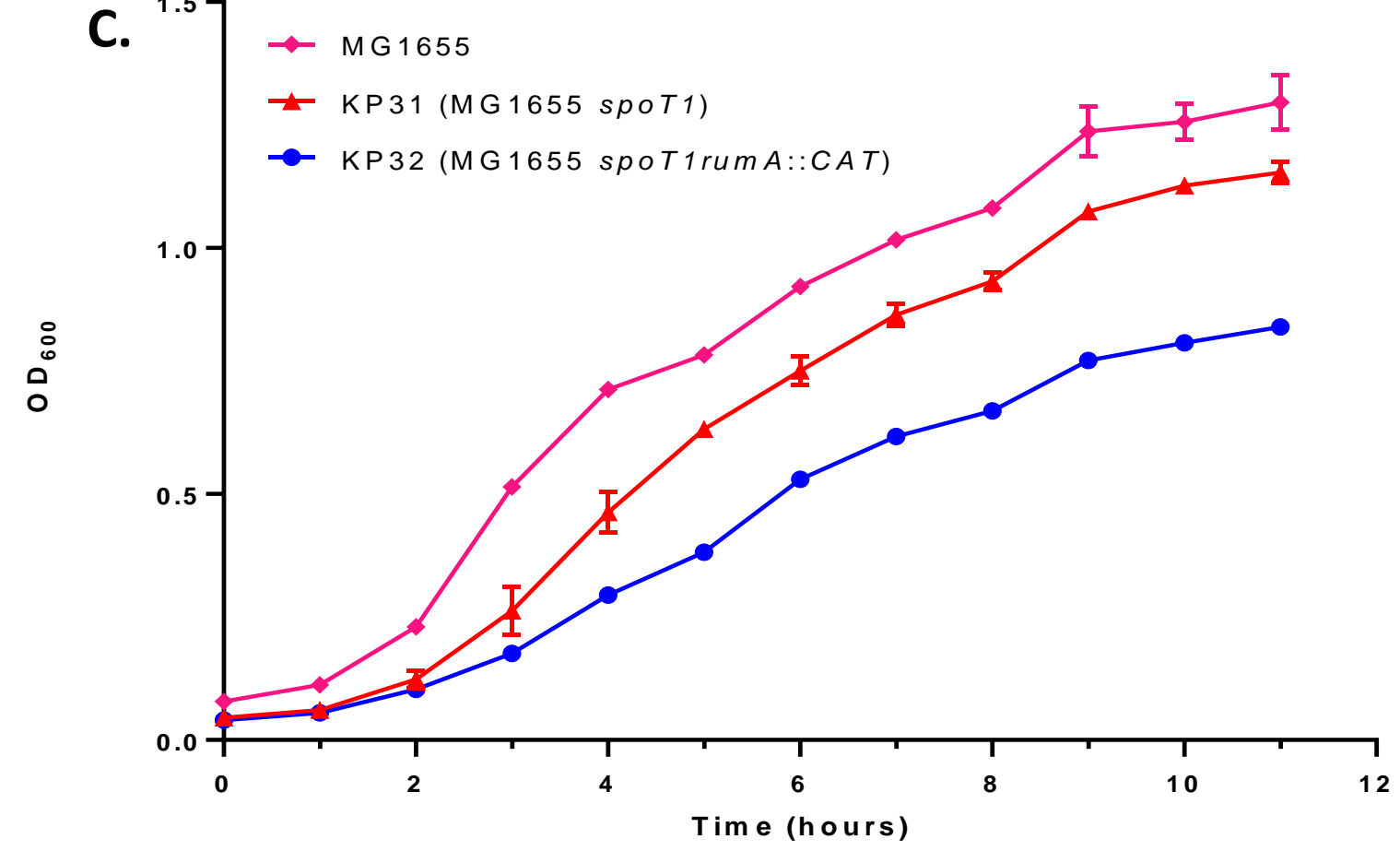

B. $\left.^{2.0}\right] \rightarrow$ MG 1655

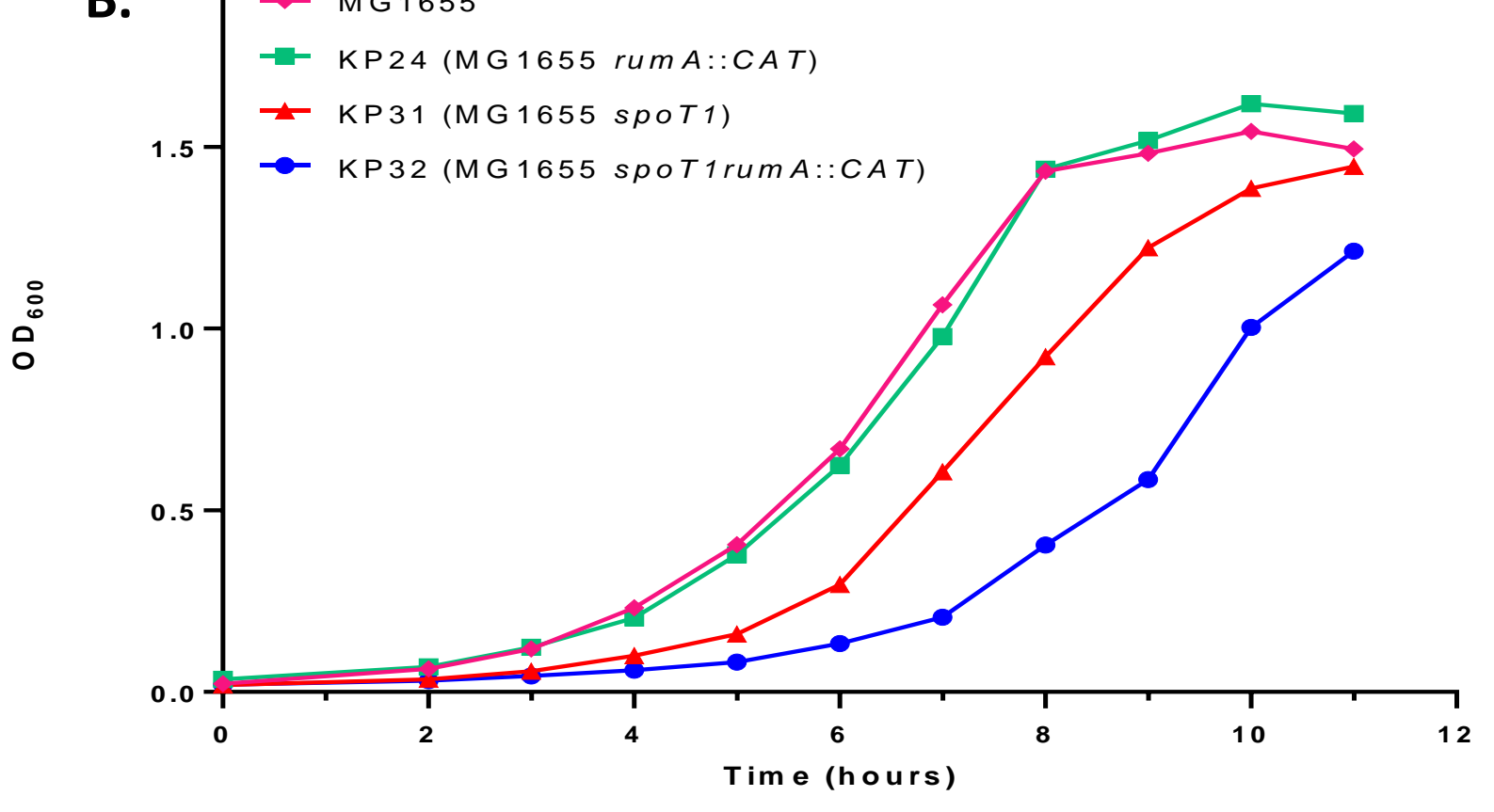

D.

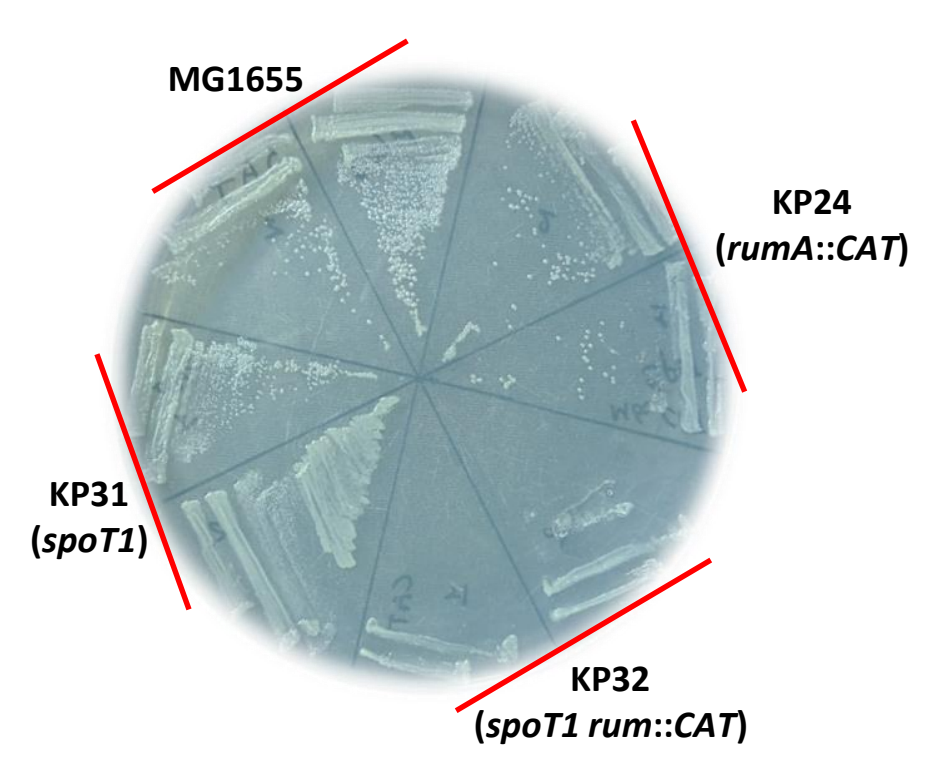


A.

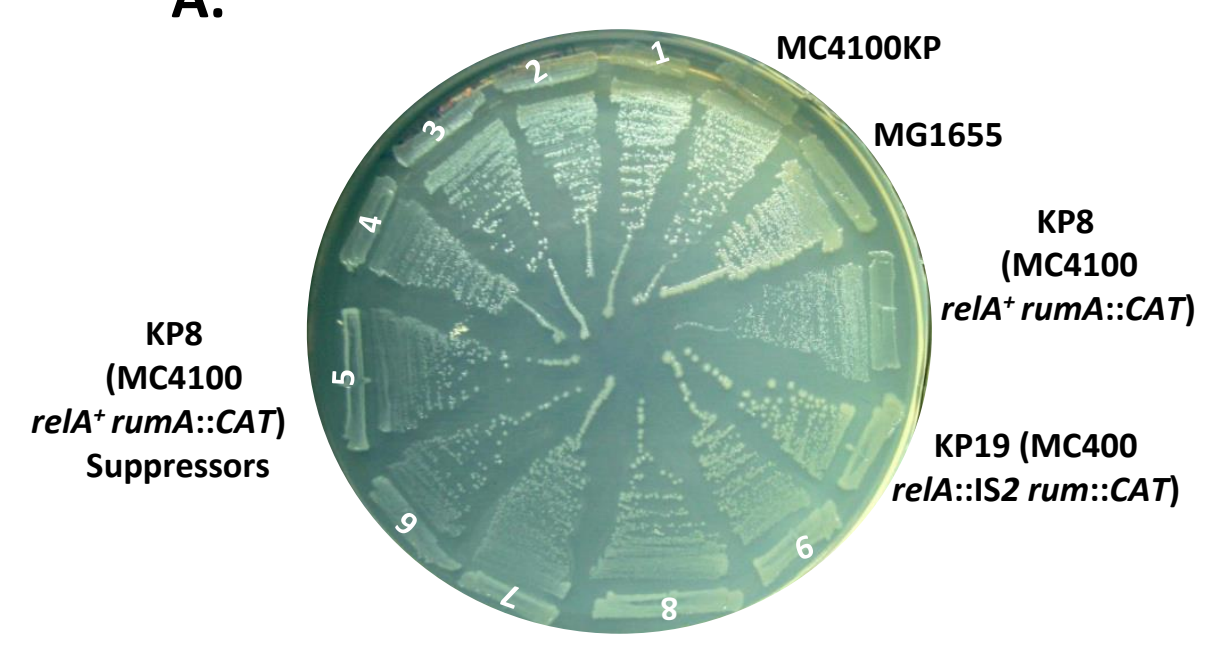

c.
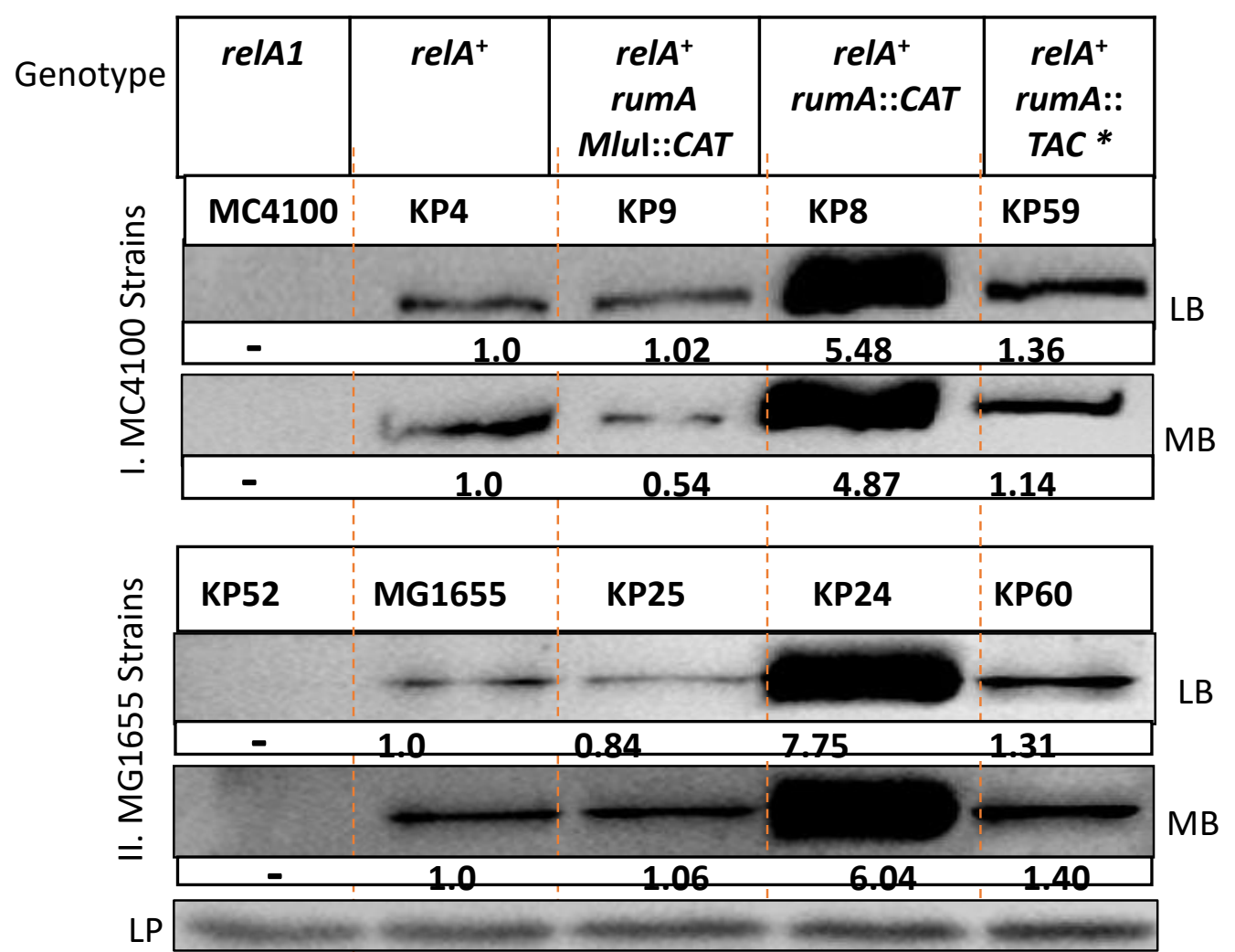

B.

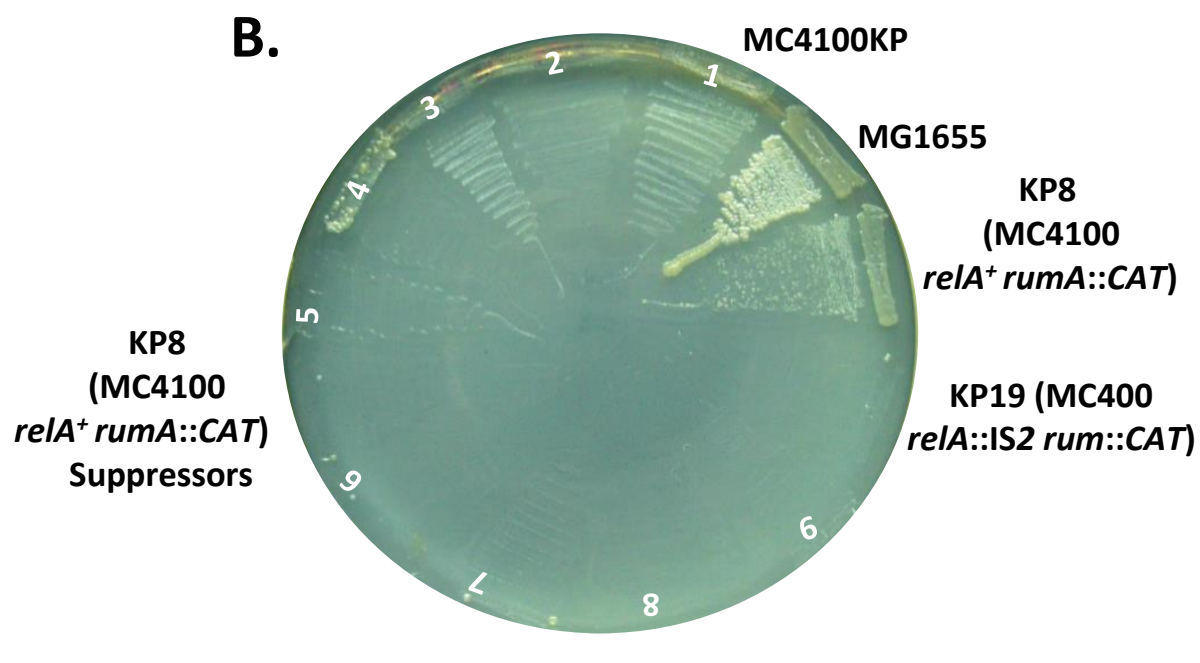

D.

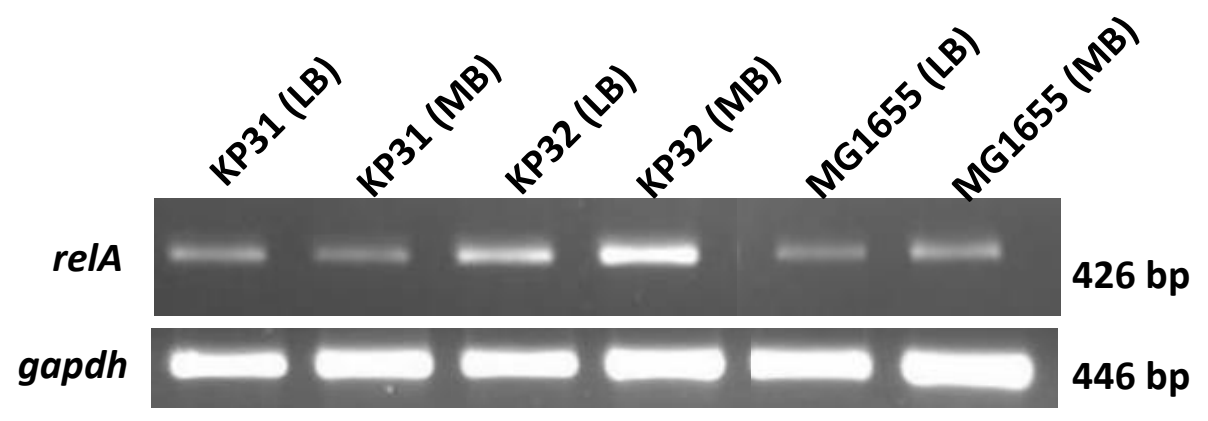


I.

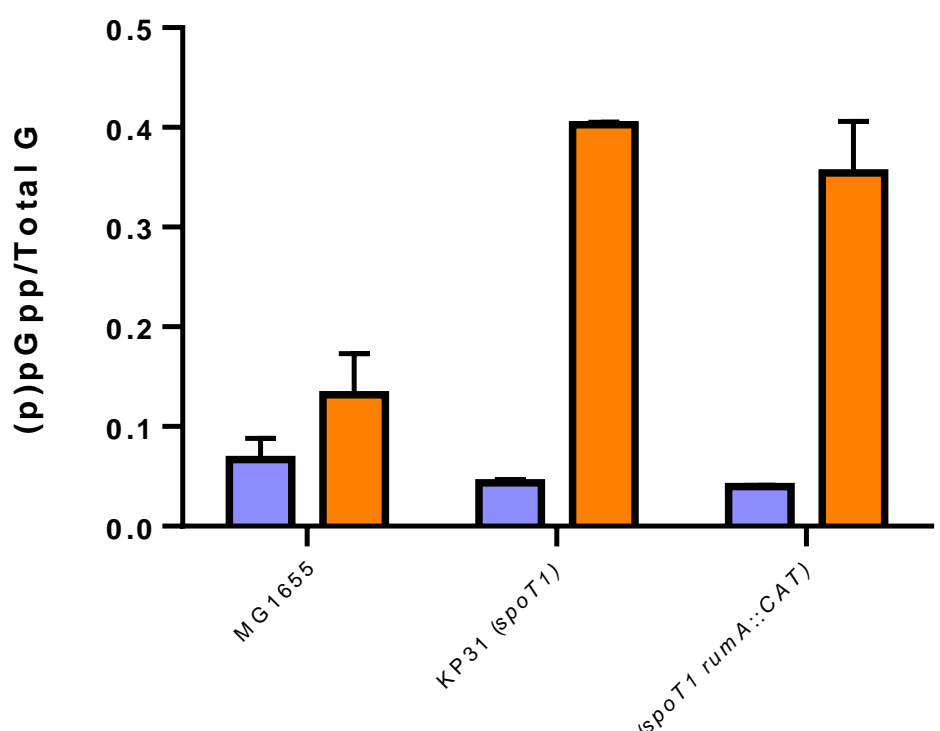

B.

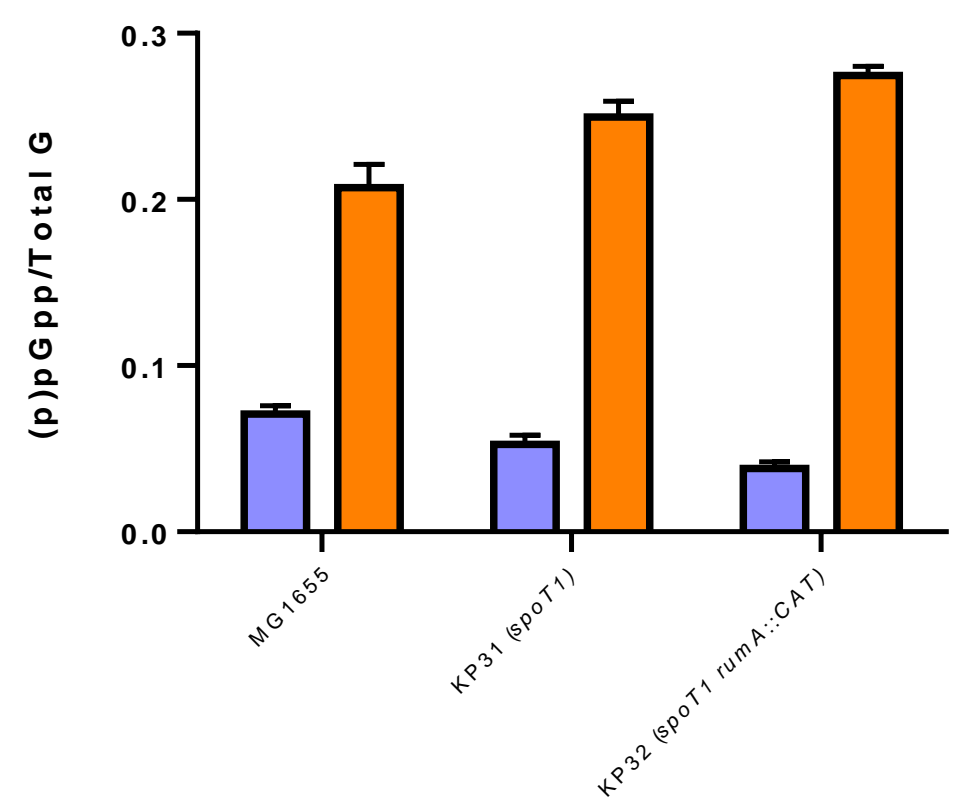

II.

KP31

KP32

MG1655
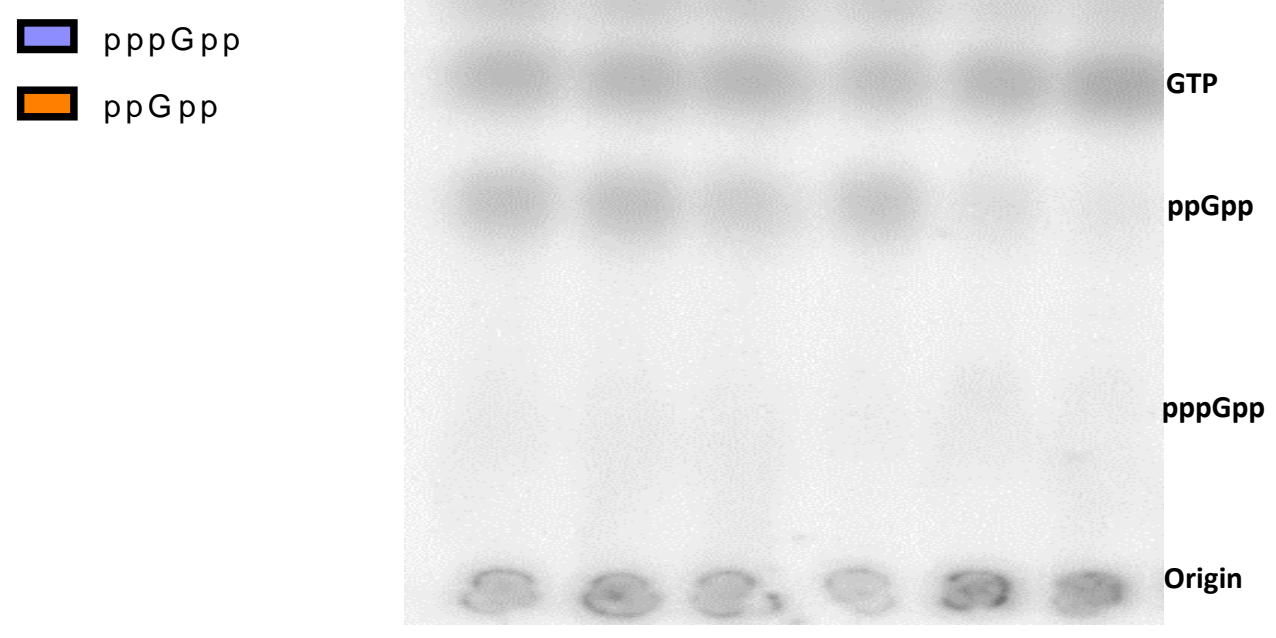

c.

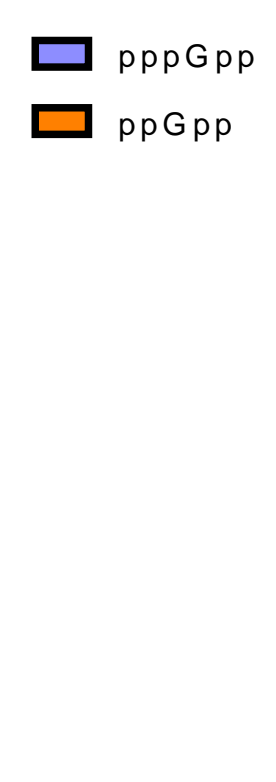

$\square$ pppGpp

$\square$ ppGpp

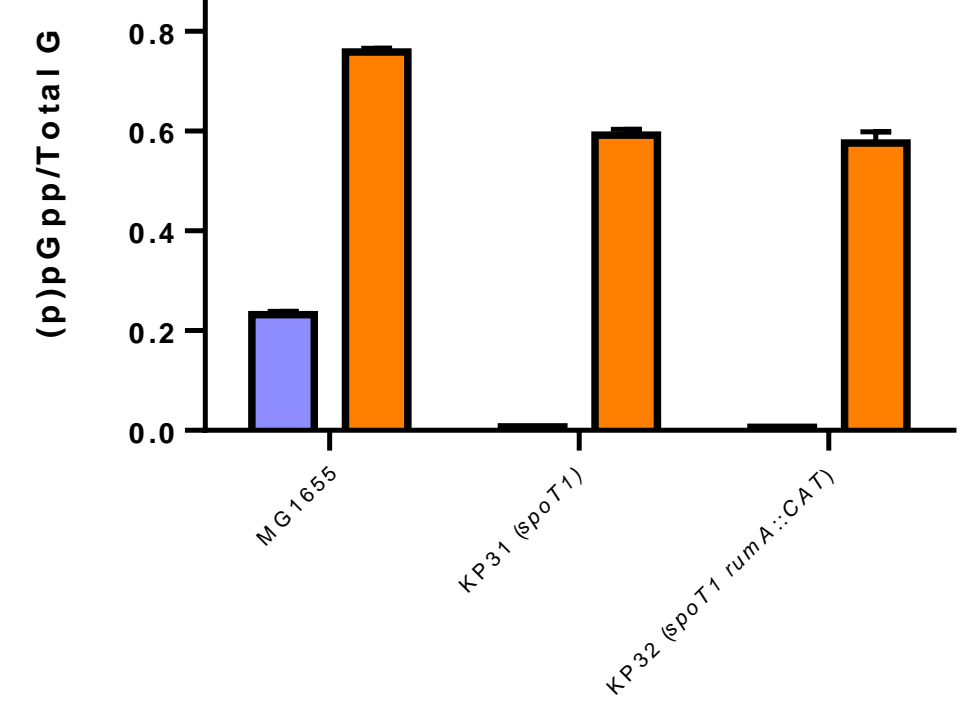

GTP

ppGpp

pppGpp

Origin 
Ic Minimal medium + 3-AT

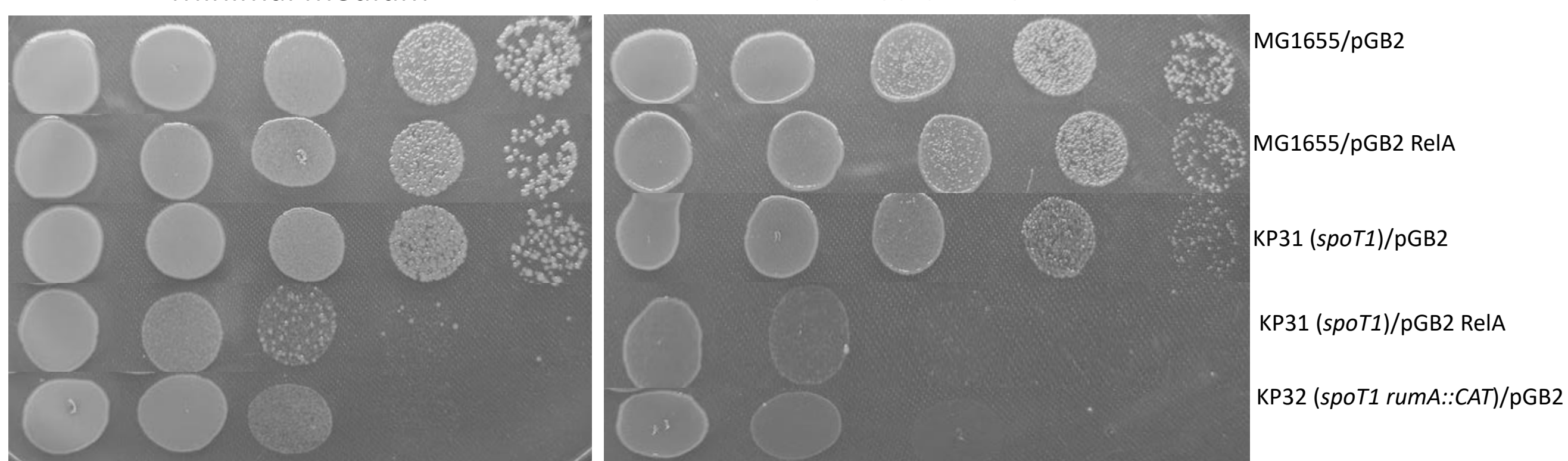

Ib

- MG1655/pGB2 ReIA

- KP31(sPoT1)/PGB2

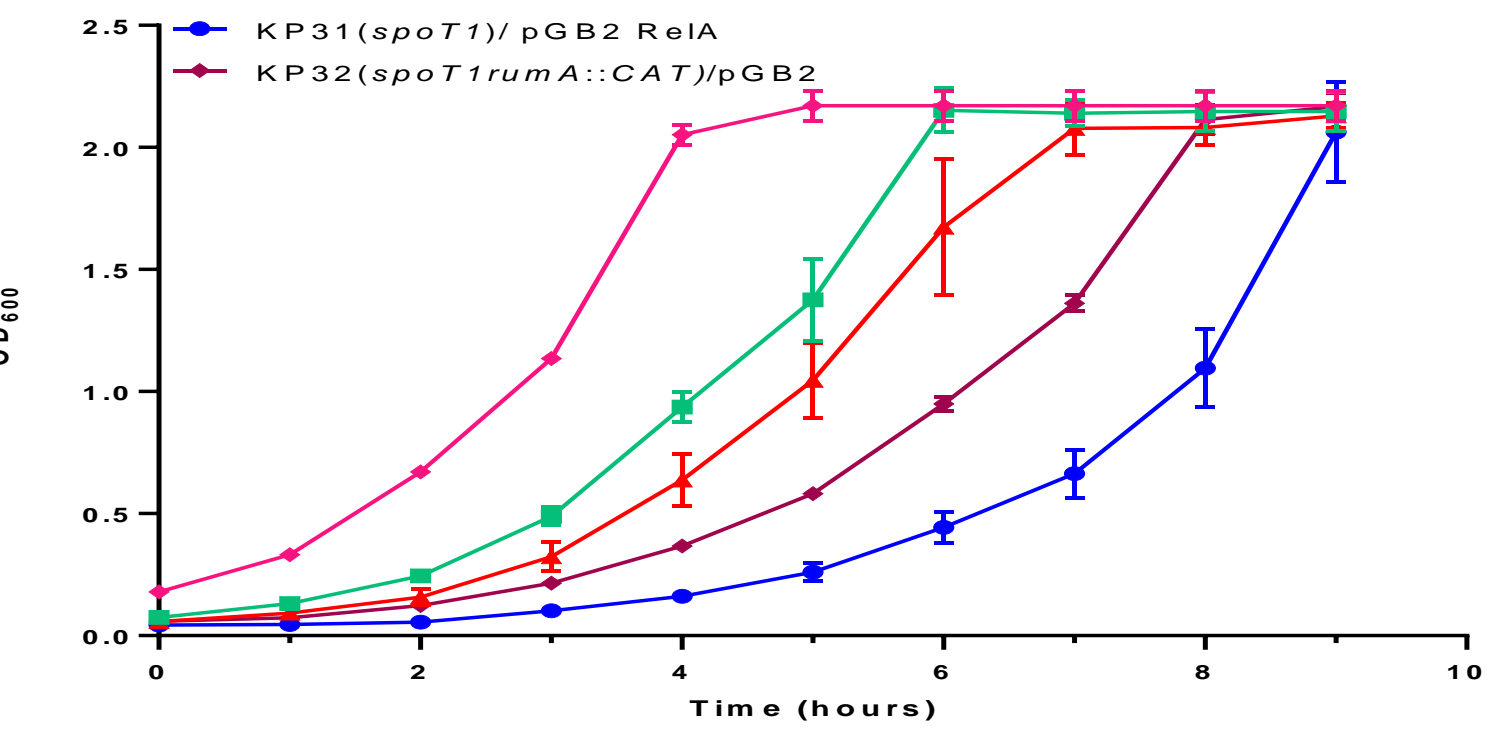




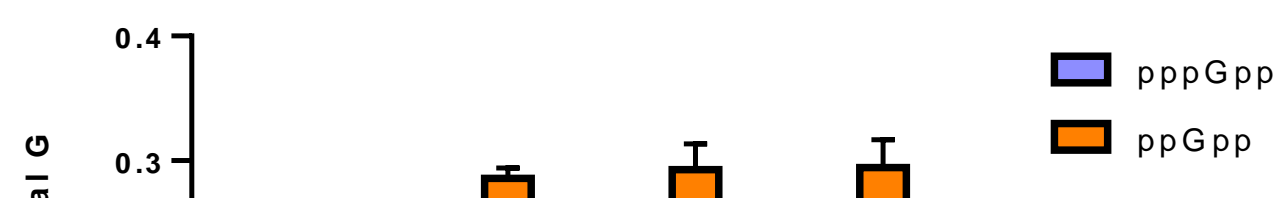

pppGpp

$\frac{0}{0}$
$\frac{0}{0}$
$\frac{1}{2}$
0
0
$\frac{2}{2}$

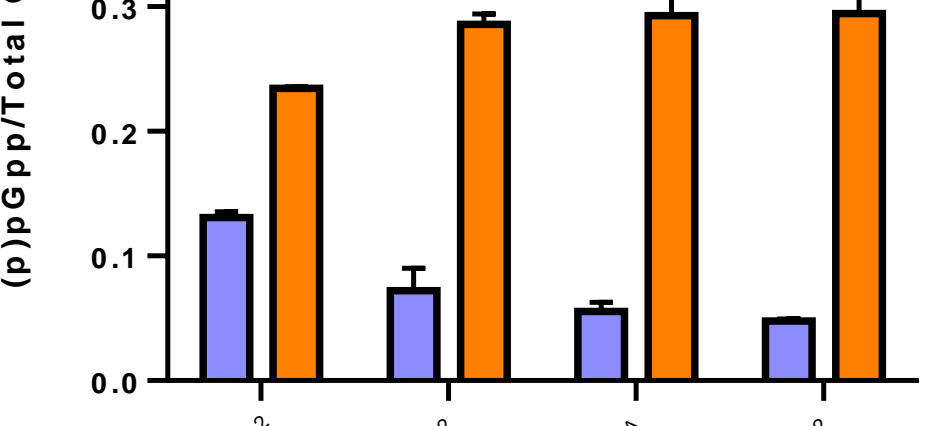

Protein Expression

30090000 on
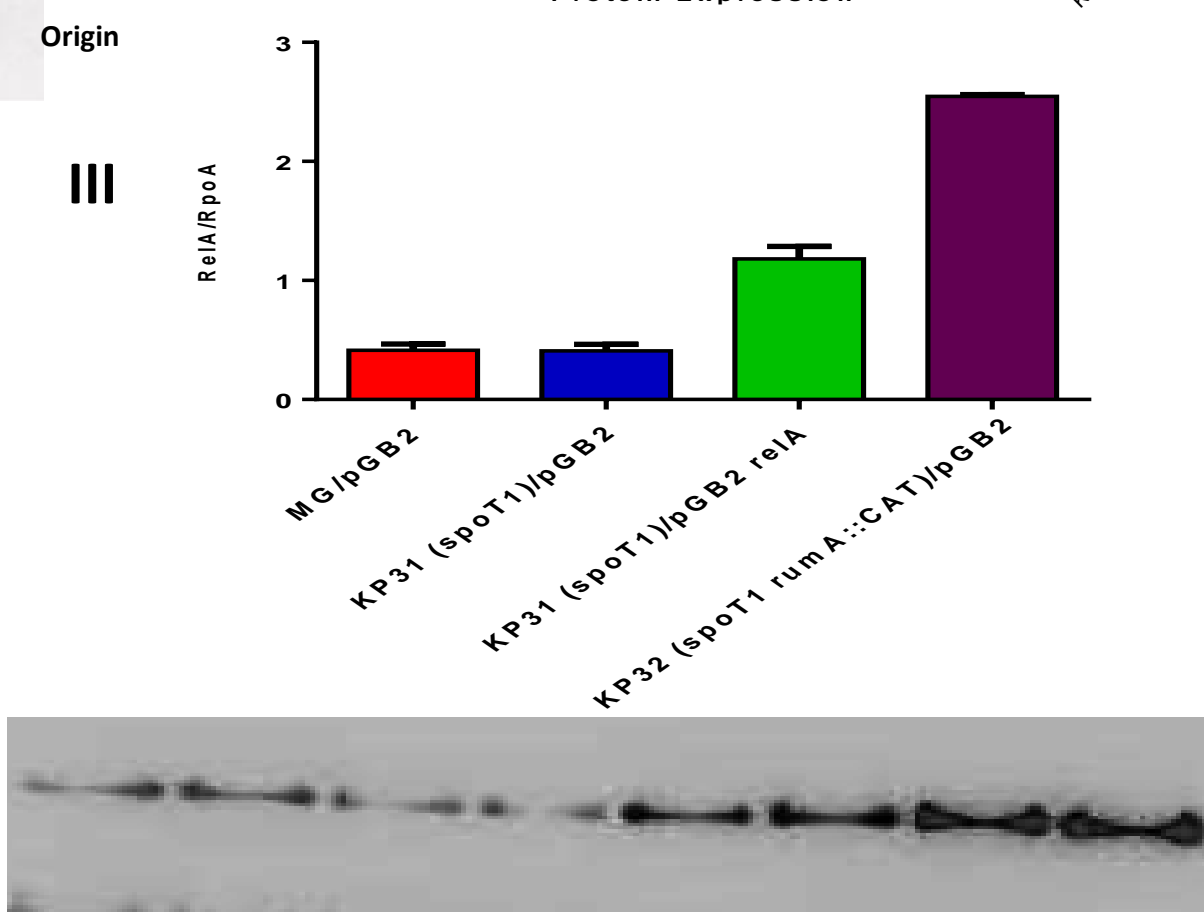

RelA 
Fig. 5B

MG1655 $\left(\right.$ relA $\left.A^{+}\right)$derivatives

la

$10^{-2} \quad 10^{-3}$

$10^{-4} \quad 10^{-5} \quad 10^{-1}$
IC

$\begin{array}{lll}10^{-3} & 10^{-4} & 10^{-5}\end{array}$ Minimal medium + 3AT

\begin{tabular}{|c|c|c|c|}
\hline Strains/plasmid & Minimal medium & Minimal medium + 3AT & \\
\hline MG1655pBAD18Kan & & & $-\frac{1}{2}$ \\
\hline \multicolumn{4}{|l|}{ KP31 (spoT1) pBAD18Kan } \\
\hline \multicolumn{4}{|l|}{ MG1655/pTE6 } \\
\hline \multicolumn{4}{|l|}{ KP31 (spoT1)/pTE6 } \\
\hline \multicolumn{4}{|l|}{ MG1655/pTE22 } \\
\hline \multicolumn{4}{|l|}{ KP31 (spoT1)/pTE22 } \\
\hline KP31 (spoT1) pTE23 & & & \\
\hline
\end{tabular}

Ib

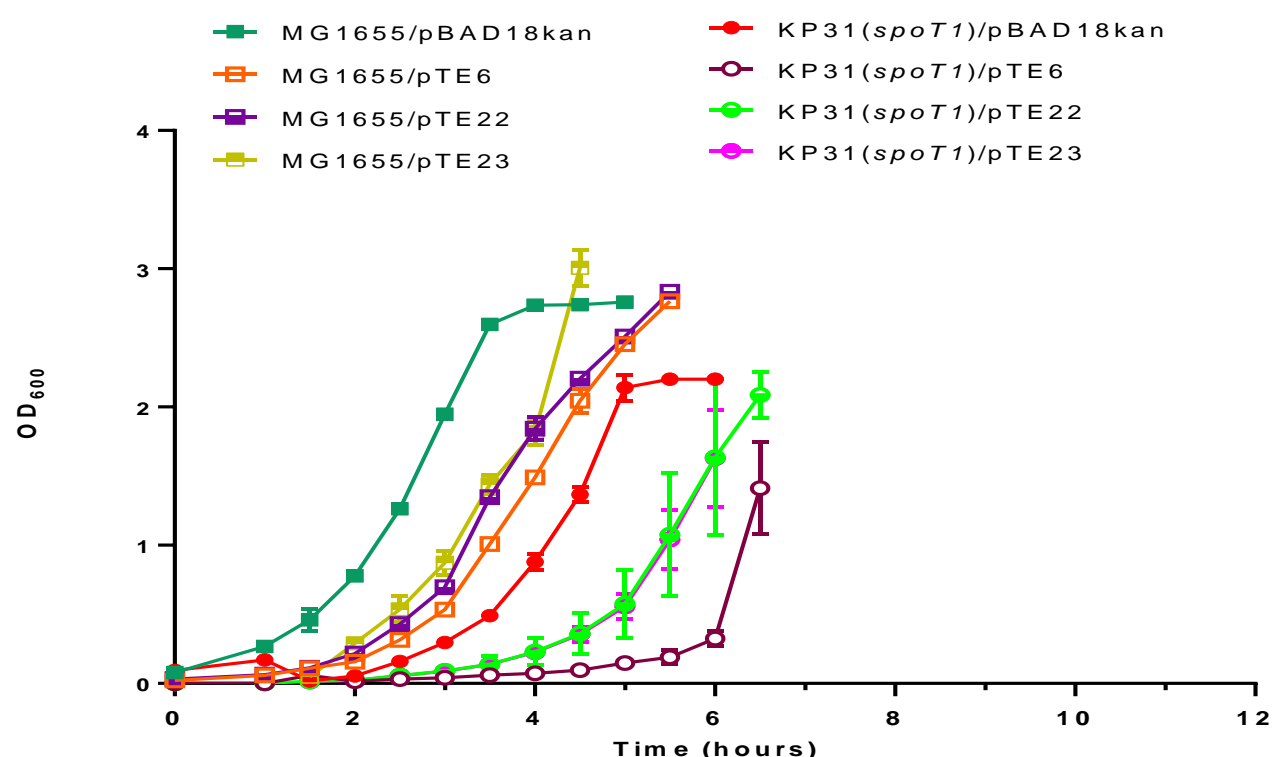

Fig. 5C

MG1655/pALS10

KP32 (spoT1 rumA::CAT)/pALS14

MG1655/pALS13

KP31 (spoT1) /pALS14

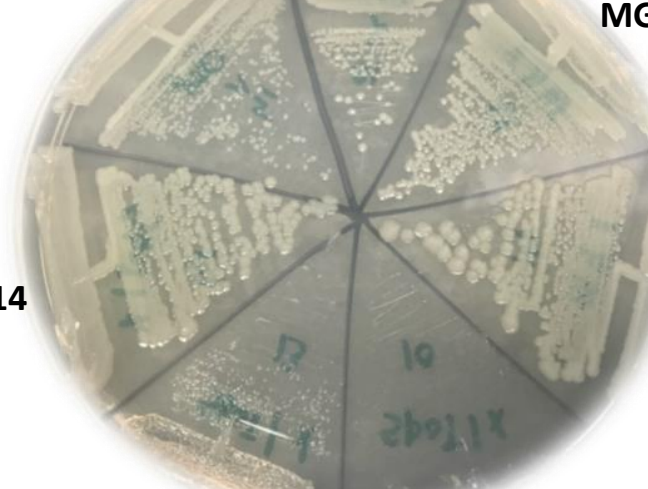

MG1655/pALS14 
Fig. 6

\section{MG1655 (relA1 ) derivatives}

la

IC

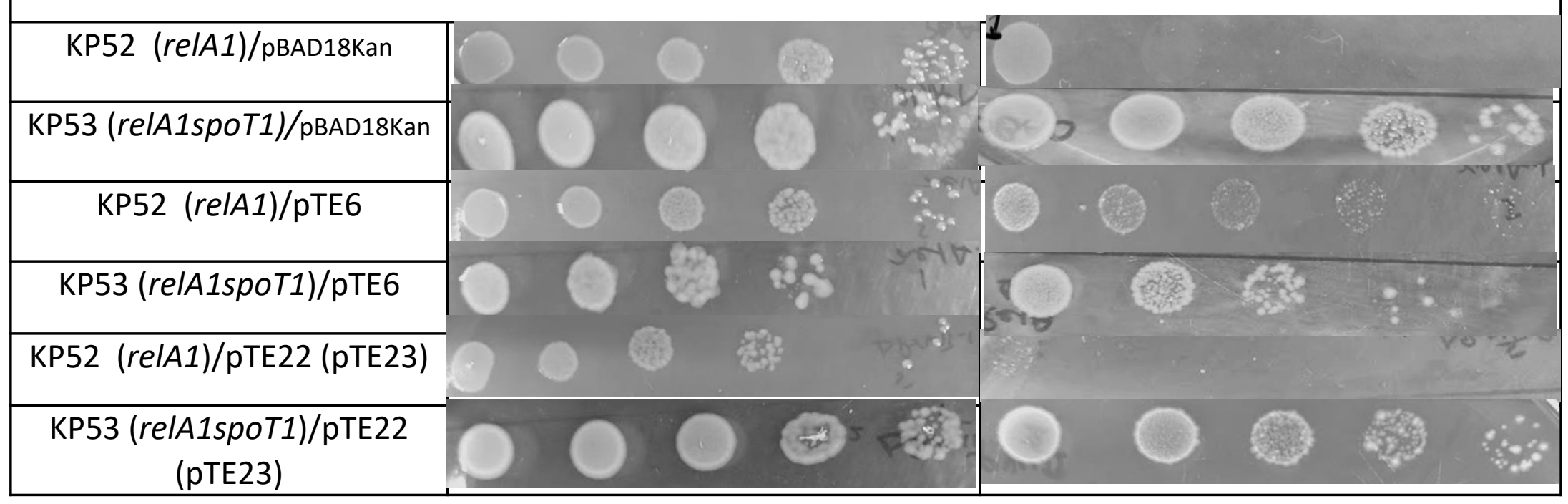

Ib

KP52(MG rela 1)/pBAD18kan

$\leadsto$ KP52(MG relA 1)/PTE6

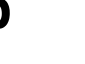

$\leadsto$ KP52(MG reIA 1)/PTE22

- KP52(MG reIA1)/PTE23 $\triangle \mathrm{KP} 53(\mathrm{MGre} / \mathrm{A} 1$ spot1)/pBAD $18 \mathrm{kan}$

$\triangle$ KP53(MG relA1spoT1)/PTE 6

$\multimap$ KP53(MG rela1spot1)/pTE22

$\leadsto$ KP53(MG rela1spoT1)/pTE23

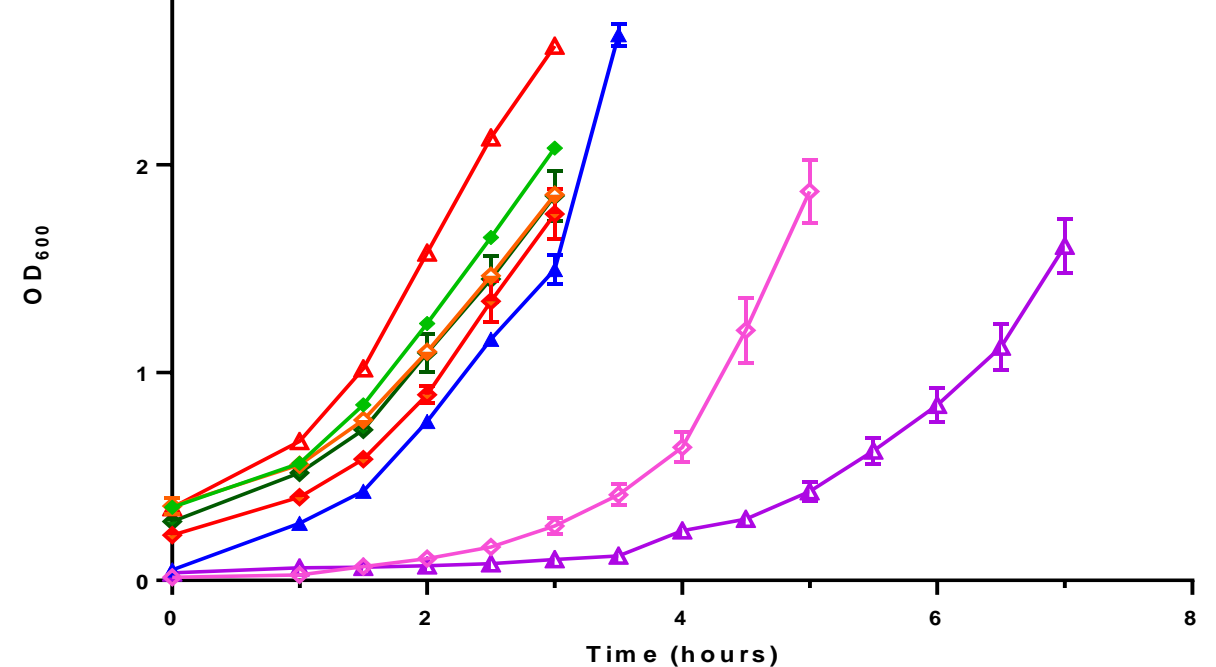


Ila KP56 (spoT202 relA1)

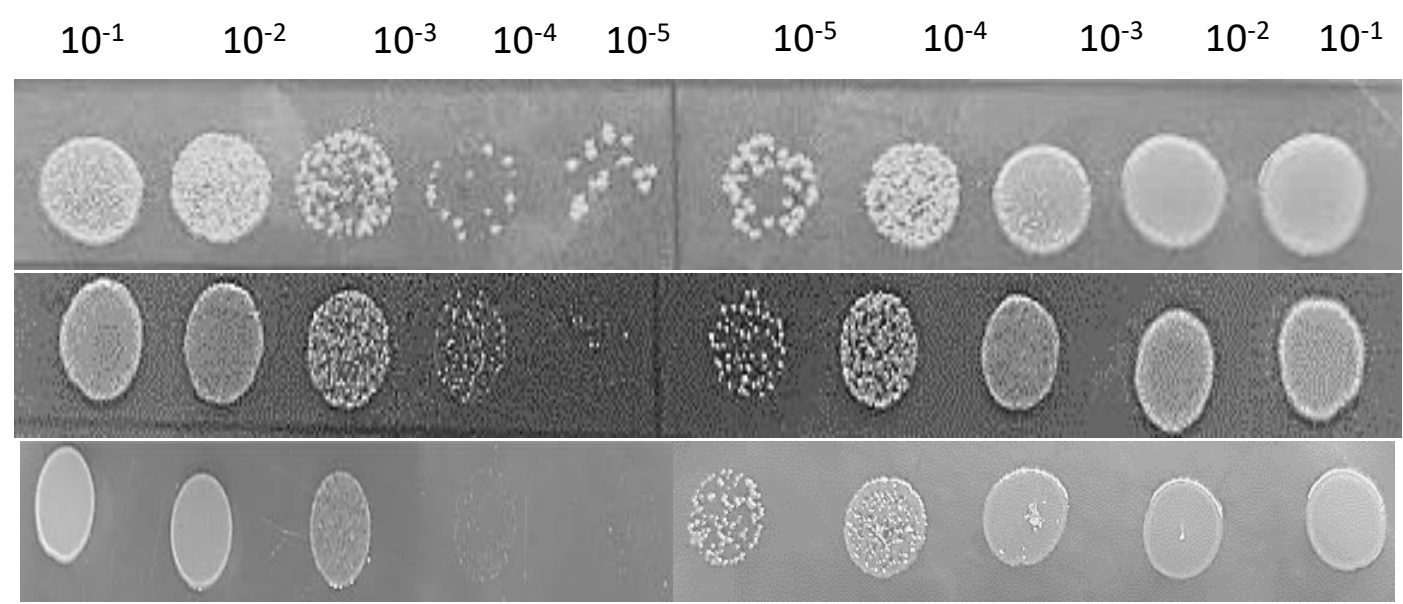

IIb

$\rightarrow$ KP57(MG relA 1)/pBAD $18 \mathrm{Kan}$

- KP57(MG reIA 1)/PTE6

- KP57(MG reIA 1)/PTE22

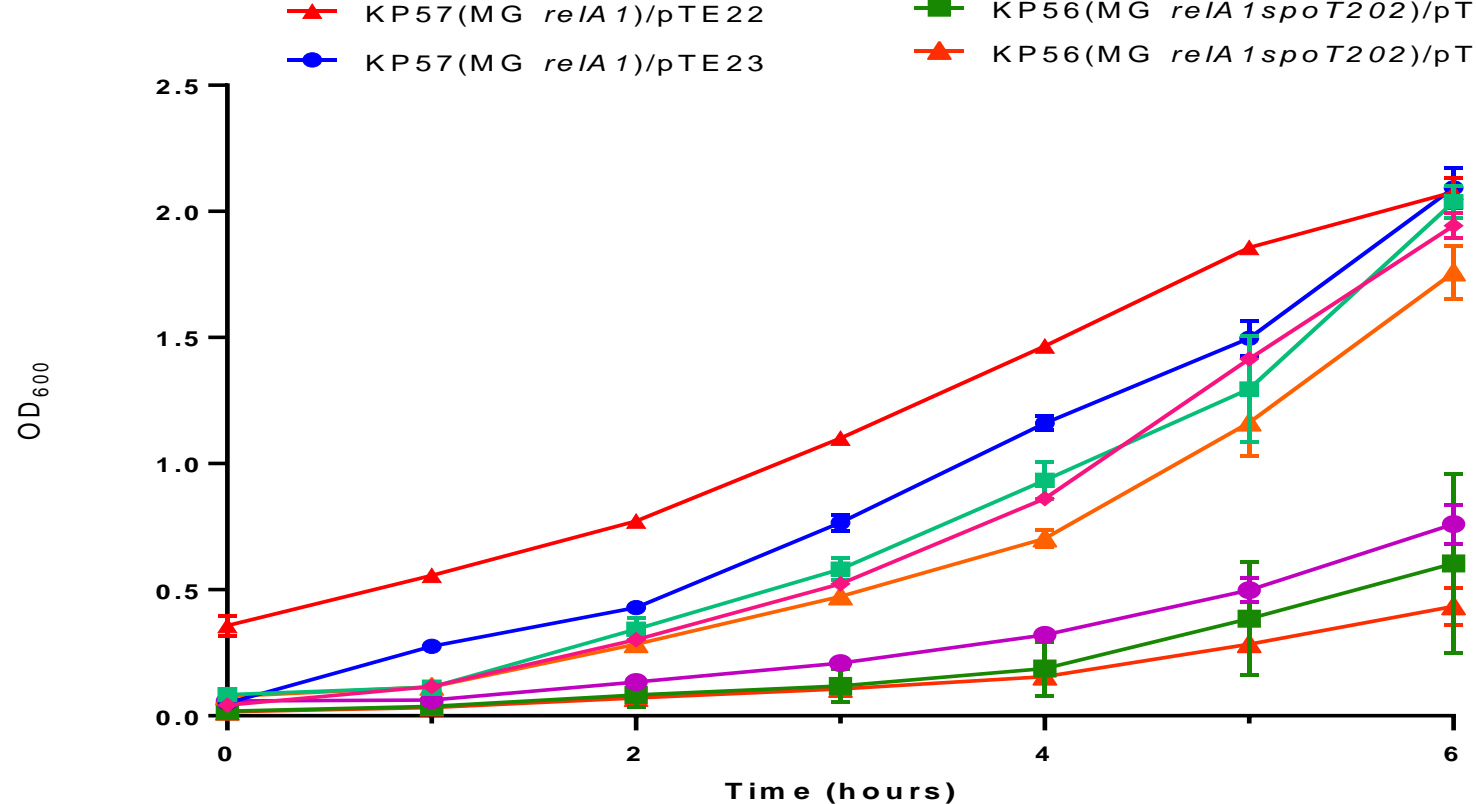

- KP56(MG relA1spoT202)/pBAD $18 \mathrm{Kan}$

- KP56(MG reIA 1 spoT2O2)/PTE 6
- KP56(MG reIA 1 spoT202)/pTE 22
pBAD18kan

pTE22 (RelA-CTD1)

pTE23 (RelA-CTD2)
C. I.

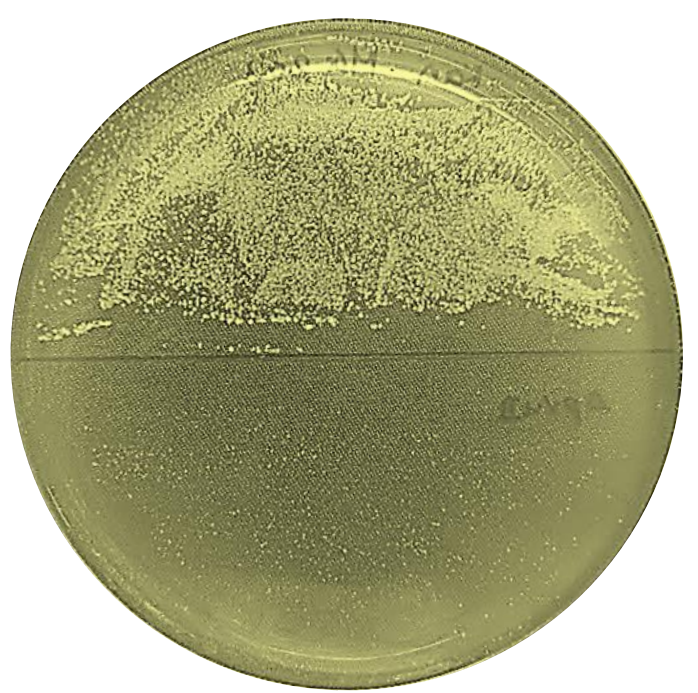

II.

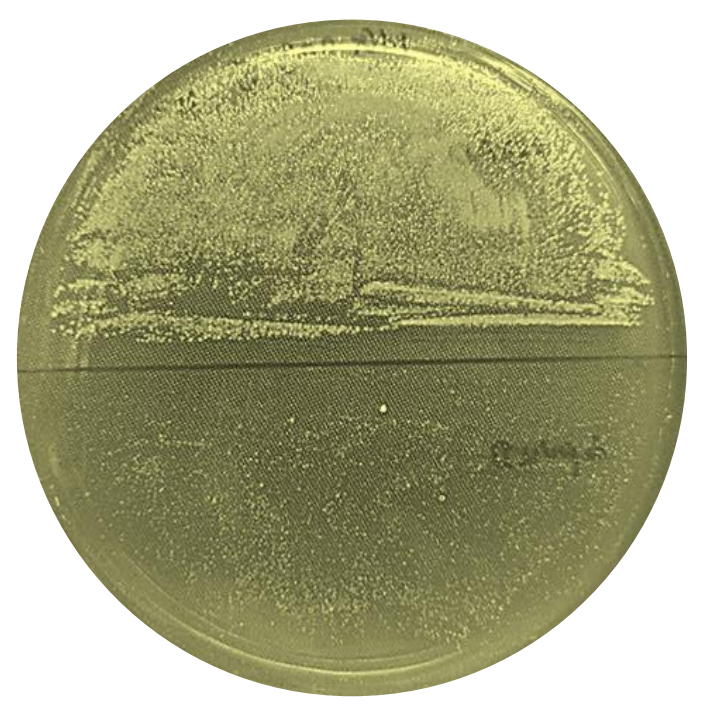

